\title{
Scalar curvature rigidity with a volume constraint
}

\author{
Pengzi Miao and Luen-Fai Tam
}

\begin{abstract}
Motivated by Brendle-Marques-Neves' counterexample to the Min-Oo's conjecture, we prove a volume constrained scalar curvature rigidity theorem which applies to the hemisphere.
\end{abstract}

\section{Introduction}

Recently, Brendle, Marques and Neves [6] have solved the long-standing Min-Oo's conjecture [15] by constructing a counterexample.

Theorem 1.1 (Brendle, Marques and Neves [6]). Suppose $n \geq 3$. Let $\bar{g}$ be the standard metric on the hemisphere $\mathbb{S}_{+}^{n}$. There exists a smooth metric $g$ on $\mathbb{S}_{+}^{n}$, which can be made to be arbitrarily close to $\bar{g}$ in the $C^{\infty}$-topology, satisfying

- the scalar curvature of $g$ is at least that of $\bar{g}$ at each point in $\mathbb{S}_{+}^{n}$,

- $g$ and $\bar{g}$ agree in a neighborhood of $\partial \mathbb{S}_{+}^{n}$,

but $g$ is not isometric to $\bar{g}$.

In this paper, we observe that if the metric $g$ in Theorem 1.1 is assumed to satisfy an additional volume constraint, then it must be isometric to $\bar{g}$. Precisely, we have

Theorem 1.2. Let $\bar{g}$ be the standard metric on $\mathbb{S}_{+}^{n}$. Let $g$ be another metric on $\mathbb{S}_{+}^{n}$ with the properties

- $R(g) \geq R(\bar{g})$ in $\mathbb{S}_{+}^{n}$,

- $H(g) \geq H(\bar{g})$ on $\partial \mathbb{S}_{+}^{n}$,

- $g$ and $\bar{g}$ induce the same metric on $\partial \mathbb{S}_{+}^{n}$,

where $R(g), R(\bar{g})$ are the scalar curvature of $g, \bar{g}$, and $H(g), H(\bar{g})$ are the mean curvature of $\partial \mathbb{S}_{+}^{n}$ in $\left(\mathbb{S}_{+}^{n}, g\right),\left(\mathbb{S}_{+}^{n}, \bar{g}\right)$. Suppose in addition

$$
V(g) \geq V(\bar{g}),
$$


where $V(g), V(\bar{g})$ are the volume of $g, \bar{g}$. If $\|g-\bar{g}\|_{C^{2}\left(\overline{\mathbb{S}}_{+}^{n}\right)}$ is sufficiently small, then there is a diffeomorphism $\varphi: \mathbb{S}_{+}^{n} \rightarrow \mathbb{S}_{+}^{n}$ with $\left.\varphi\right|_{\partial \mathbb{S}_{+}^{n}}=\mathrm{id}$, the identify map on $\partial \mathbb{S}_{+}^{n}$, such that $\varphi^{*}(g)=\bar{g}$.

Theorem 1.2 is indeed a special case of a more general result:

Theorem 1.3. Let $(\Omega, \bar{g})$ be an $n$-dimensional compact Riemannian manifold, of constant sectional curvature 1 , with smooth boundary $\Sigma$. Suppose $\overline{\mathbb{I I}}+\bar{H} \bar{\gamma} \geq 0$ (i.e., $\overline{\mathbb{I I}}+\bar{H} \bar{\gamma}$ is positive semi-definite), where $\bar{\gamma}$ is the induced metric on $\Sigma$ and $\overline{\mathbb{I I}}, \bar{H}$ are the second fundamental form, the mean curvature of $\Sigma$ in $(\Omega, \bar{g})$. Suppose the first nonzero Neumann eigenvalue $\mu$ of $(\Omega, \bar{g})$ satisfies $\mu>n-\frac{2}{n+1}$.

Consider a nearby metric $g$ on $\Omega$ with the properties

- $R(g) \geq n(n-1)$ where $R(g)$ is the scalar curvature of $g$,

- $H(g) \geq \bar{H}$ where $H(g)$ is the mean curvature of $\Sigma$ in $(\Omega, g)$,

- $g$ and $\bar{g}$ induce the same metric on $\Sigma$,

- $V(g) \geq V(\bar{g})$ where $V(g), V(\bar{g})$ are the volumes of $g, \bar{g}$.

If $\|g-\bar{g}\|_{C^{2}(\bar{\Omega})}$ is sufficiently small, then there is a diffeomorphism $\varphi$ on $\Omega$ with $\left.\varphi\right|_{\Sigma}=\mathrm{id}$, such that $\varphi^{*}(g)=\bar{g}$.

As a by-product of the method used to derive Theorem 1.3, we obtain a volume estimate for metrics close to the Euclidean metric in terms of the scalar curvature.

Theorem 1.4. Let $\Omega \subset \mathbb{R}^{n}$ be a bounded domain with smooth boundary $\Sigma$. Suppose $\overline{\mathbb{I I}}+\bar{H} \bar{\gamma}>0$ (i.e., $\overline{\mathbb{I I}}+\bar{H} \bar{\gamma}$ is positive definite), where $\overline{\mathbb{I I I}}, \bar{H}$ are the second fundamental form, the mean curvature of $\Sigma$ in $\mathbb{R}^{n}$ and $\bar{\gamma}$ is the metric on $\Sigma$ induced from the Euclidean metric $\bar{g}$. Let $g$ be another metric on $\bar{\Omega}$ satisfying

- $H(g) \geq \bar{H}$, where $H(g)$ is the mean curvature of $\Sigma$ in $(\Omega, g)$

- $g$ and $\bar{g}$ induce the same metric on $\Sigma$.

Given any point $a \in \mathbb{R}^{n}$, there exists a constant $\Lambda>\frac{\max _{q \in \bar{\Omega}}|q-a|^{2}}{4(n-1)}$, depending only on $\Omega$ and $a$, such that if $\|g-\bar{g}\|_{C^{3}(\bar{\Omega})}$ is sufficiently small, then

$$
V(g)-V(\bar{g}) \geq \int_{\Omega} R(g) \Phi d \operatorname{vol}_{\bar{g}},
$$

where $\Phi(x)=-\frac{1}{4(n-1)}|x-a|^{2}+\Lambda>0$ on $\bar{\Omega}$. 
Theorem 1.4 may be compared to a previous theorem of Bartnik [2], which estimates the total mass [1] of an asymptotically flat metric that is a perturbation of the Euclidean metric.

Theorem 1.5 (Bartnik [2]). Let $g$ be an asymptotically flat metric on $\mathbb{R}^{3}$. If $g$ is sufficiently close to the Euclidean metric $\bar{g}$ (in certain weighted Sobolev space), then

$$
16 \pi \mathfrak{m}(g) \geq \int_{\mathbb{R}^{3}} R(g) d \operatorname{vol}_{\bar{g}}
$$

where $\mathfrak{m}(g)$ is the total mass of $g$.

Our proofs of Theorems 1.2-1.4 follow a recent perturbation analysis of Brendle and Marques in [5], where they established a scalar curvature rigidity theorem for "small" geodesic balls in $\mathbb{S}^{n}$.

Theorem 1.6 (Brendle and Marques [5]). Let $\Omega \subset \mathbb{S}^{n}$ be a geodesic ball of radius $\delta$. Suppose

$$
\cos \delta \geq \frac{2}{\sqrt{n+3}}
$$

Let $\bar{g}$ be the standard metric on $\mathbb{S}^{n}$. Let $g$ be another metric on $\Omega$ with the properties

- $R(g) \geq n(n-1)$ at each point in $\Omega$,

- $H(g) \geq \bar{H}$ at each point on $\partial \Omega$,

- $g$ and $\bar{g}$ induce the same metric on $\partial \Omega$,

where $R(g)$ is the scalar curvature of $g$, and $H(g), \bar{H}$ are the mean curvature of $\partial \Omega$ in $(\Omega, g),(\Omega, \bar{g})$. If $g-\bar{g}$ is sufficiently small in the $C^{2}$-norm, then $\varphi^{*}(g)=\bar{g}$ for some diffeomorphism $\varphi: \Omega \rightarrow \Omega$ such that $\left.\varphi\right|_{\partial \Omega}=\mathrm{id}$.

In Theorem 1.6, the condition (1.3) is equivalently to

$$
\bar{H} \geq 4 \tan \delta
$$

because the mean curvature $\bar{H}$ of $\partial B(\delta)$ is $(n-1) \frac{\cos \delta}{\sin \delta}$. As another application of the formulas in Section 2, we obtain a generalization of Theorem 1.6 to convex domains in $\mathbb{S}^{n}$. 
Theorem 1.7. Let $\Omega \subset \mathbb{S}^{n}$ be a smooth domain contained in a geodesic ball $B$ of radius less than $\frac{\pi}{2}$. Let $\bar{g}$ be the standard metric on $\mathbb{S}^{n}$. Let $\overline{\mathbb{I I}}, \bar{H}$ be the second fundamental form, the mean curvature of $\partial \Omega$ in $(\Omega, \bar{g})$. Suppose $\Omega$ is convex, i.e., $\overline{\mathbb{I I}} \geq 0$. At $\partial \Omega$, suppose

$$
\bar{H} \geq 4 \tan r,
$$

where $r$ is the $\bar{g}$-distance to the center of $B$. Then the conclusion of Theorem 1.6 holds on $\Omega$.

Theorem 1.7 is an immediate corollary of Theorem 5.1 in Section 5. In a simpler setting, where the background metric $\bar{g}$ is a flat metric, we have

Theorem 1.8. Let $\Omega$ be a compact manifold with smooth boundary $\Sigma$. Suppose there is a flat metric $\bar{g}$ on $\Omega$ such that $\overline{\mathbb{I I}}+\bar{H} \bar{\gamma} \geq 0$ (i.e., $\overline{\mathbb{I I}}+\bar{H} \bar{\gamma}$ is positive semi-definite), where $\overline{\mathbb{I I}}, \bar{H}$ are the second fundamental form, the mean curvature of $\Sigma$, and $\bar{\gamma}$ is the induced metric on $\Sigma$. Given another metric $g$ on $\Omega$ such that

- $R(g) \geq 0$ on $\Omega$,

- $H(g) \geq \bar{H}$ at $\Sigma$,

- $g$ and $\bar{g}$ induce the same metric on $\Sigma$,

if $\|g-\bar{g}\|_{C^{2}(\bar{\Omega})}$ is sufficiently small, then $\varphi^{*}(g)=\bar{g}$ for some diffeomorphism $\varphi: \Omega \rightarrow \Omega$ with $\left.\varphi\right|_{\Sigma}=\mathrm{id}$.

Similar calculation at the infinitesimal level provides examples of compact 3-manifolds of nonnegative scalar curvature whose boundary surface does not have positive Gaussian curvature but still has positive BrownYork mass $[7,8]$. We include this in the end of the paper to compare with known results in [17].

Theorem 1.9. Let $\Sigma \subset \mathbb{R}^{n}$ be a connected, closed hypersurface satisfying $\overline{\mathbb{I I}}+\bar{H} \bar{\gamma} \geq 0$, where $\overline{\mathbb{I I}}, \bar{H}$ are the second fundamental form, the mean curvature of $\Sigma$, and $\bar{\gamma}$ is the induced metric on $\Sigma$. Let $\Omega$ be the domain enclosed by $\Sigma$ in $\mathbb{R}^{n}$. Let $h$ be any nontrivial $(0,2)$ symmetric tensor on $\Omega$ satisfying

$$
\operatorname{div}_{\bar{g}} h=0, \quad \operatorname{tr}_{\bar{g}} h=0,\left.\quad h\right|_{T \Sigma}=0 .
$$

Let $\{g(t)\}_{|t|<\epsilon}$ be a 1-parameter family of metrics on $\Omega$ satisfying

$$
g(0)=\bar{g}, \quad g^{\prime}(0)=h, \quad R(g(t)) \geq 0,\left.\quad g(t)\right|_{T \Sigma}=\left.\bar{g}\right|_{T \Sigma} .
$$


Then

$$
\int_{\Sigma} \bar{H} d \sigma_{\bar{g}}>\int_{\Sigma} H(g(t)) d \sigma_{\bar{g}}
$$

for small $t \neq 0$, where $H(g(t))$ is the mean curvature of $\Sigma$ in $(\Omega, g(t))$.

This paper is organized as follows. In Section 2, we derive a basic formula concerning a perturbed metric (Theorem 2.1), which corresponds to [5, Theorem 10] of Brendle and Marques. In Section 3, we prove Theorem 1.3, which implies Theorem 1.2. In Section 4, we give a proof of Theorem 1.4. In Section 5, we consider other applications of the formulas in Section 2 and prove Theorem 1.7-1.9.

\section{Basic formulas for a perturbed metric}

Let $\Omega$ be an $n$-dimensional, smooth, compact manifold with boundary $\Sigma$. Let $\bar{g}$ be a fixed smooth Riemannian metric on $\Omega$. Given a tensor $\eta$, let " $|\eta| "$ denote the length of $\eta$ measured with respect to $\bar{g}$. Denote the covariant derivative with respect to $\bar{g}$ by $\bar{\nabla}$. Indices of tensors are raised by $\bar{g}$. Let $\bar{R}_{i k j l}$ denote the curvature tensor of $\bar{g}$ such that if $\bar{g}$ has constant sectional curvature $\kappa$, then $\bar{R}_{i k j l}=\kappa\left(g_{i j} g_{k l}-g_{i l} g_{k j}\right)$. Consider a nearby Riemannian metric $g=\bar{g}+h$ where $h$ is a symmetric $(0,2)$ tensor with $|h|$ very small, say $|h| \leq \frac{1}{2}$.

The following pointwise estimates of the scalar curvature of $g$ and the mean curvature of $\Sigma$ were derived by Brendle and Marques in [5].

Proposition 2.1 (Brendle and Marques [5]). The scalar curvatures $R(g)$, $R(\bar{g})$ of the metrics $g, \bar{g}$ satisfy

$$
\begin{aligned}
& \left.\left|R(g)-R(\bar{g})+\langle\operatorname{Ric}(\bar{g}), h\rangle-\left\langle\operatorname{Ric}(\bar{g}), h^{2}\right\rangle+\frac{1}{4}\right| \bar{\nabla} h\right|^{2}-\frac{1}{2} \bar{g}^{i j} \bar{g}^{k l} \bar{g}^{p q} \bar{\nabla}_{i} h_{k p} \bar{\nabla}_{l} h_{j q} \\
& \quad+\frac{1}{4}\left|\bar{\nabla}\left(\operatorname{tr}_{\bar{g}} h\right)\right|^{2}+\bar{\nabla}_{i}\left[g^{i k} g^{j l}\left(\bar{\nabla}_{k} h_{j l}-\bar{\nabla}_{l} h_{j k}\right)\right] \mid \\
& \quad \leq C\left(|h||\bar{\nabla} h|^{2}+|h|^{3}\right),
\end{aligned}
$$

where $\operatorname{Ric}(\bar{g})$ is the Ricci curvature of $\bar{g}, h^{2}$ is the $\bar{g}$-square of $h$, i.e., $\left(h^{2}\right)_{i k}=\bar{g}^{j l} h_{i j} h_{k l},\langle\cdot, \cdot\rangle$ is taken with respect to $\bar{g}$, and $C$ is a positive constant depending only on $n$. 
Remark 2.1. If the background metric $\bar{g}$ is Ricci flat, i.e., $\bar{R}_{i k}=0$, then there will be no $|h|^{3}$ term in the above estimate. That is because

$$
R(g)=g^{i k} \bar{R}_{i k}-g^{i k} g^{l j}\left(\bar{\nabla}_{i, k} h_{j l}-\bar{\nabla}_{i, l} h_{j k}\right)+g^{i k} g^{j l} g_{p q}\left(\Gamma_{i l}^{q} \Gamma_{j k}^{p}-\Gamma_{j l}^{q} \Gamma_{i k}^{p}\right)
$$

where each term on the right, except $g^{i k} \bar{R}_{i k}$, involves derivatives of $h$.

Proposition 2.2 (Brendle and Marques [5]). Assume that $g$ and $\bar{g}$ induce the same metric on $\Sigma$, i.e., $\left.h\right|_{T \Sigma}=0$ where $T \Sigma$ is the tangent bundle of $\Sigma$. Then the mean curvatures $H(g), H(\bar{g})$ of $\Sigma$ in $(\Omega, g),(\Omega, \bar{g})$, each with respect to the outward normals, satisfy

$$
\begin{aligned}
& \mid 2[H(g)-H(\bar{g})]-\left(h(\bar{\nu}, \bar{\nu})-\frac{1}{4} h(\bar{\nu}, \bar{\nu})^{2}+\sum_{\alpha=1}^{n-1} h\left(e_{\alpha}, \bar{\nu}\right)^{2}\right) H(\bar{g}) \\
& +\left(1-\frac{1}{2} h(\bar{\nu}, \bar{\nu})\right) \sum_{\alpha=1}^{n-1}\left[2 \bar{\nabla}_{e_{\alpha}} h\left(e_{\alpha}, \bar{\nu}\right)-\bar{\nabla}_{\bar{\nu}} h\left(e_{\alpha}, e_{\alpha}\right)\right] \mid \\
& \quad \leq C\left(|h|^{2}|\bar{\nabla} h|+|h|^{3}\right),
\end{aligned}
$$

where $\left\{e_{\alpha} \mid 1 \leq \alpha \leq n-1\right\}$ is a local orthonormal frame on $\Sigma, \bar{\nu}$ is the $\bar{g}$ unit outward normal vector to $\Sigma$, and $C$ is a positive constant depending only on $n$.

To derive the main formula (2.23) in this section, we let

$$
D R_{\bar{g}}(h)=-\Delta_{\bar{g}}\left(\operatorname{tr}_{\bar{g}} h\right)+\operatorname{div}_{\bar{g}} \operatorname{div}_{\bar{g}} h-\langle\operatorname{Ric}(\bar{g}), h\rangle
$$

be the linearization of the scalar curvature at $\bar{g}$ along $h$. Here " $\Delta_{\bar{g}}$, $\operatorname{div}_{\bar{g}}$ " denote the Laplacian, the divergence with respect to $\bar{g}$.

Lemma 2.1. With the same notations in Proposition 2.1, assume in addition $\operatorname{div}_{\bar{g}} h=0$, then

$$
\begin{aligned}
R(g)-R(\bar{g})= & D R_{\bar{g}}(h)-\frac{1}{2} D R_{\bar{g}}\left(h^{2}\right)+\left\langle h, \bar{\nabla}^{2} \operatorname{tr}_{\bar{g}} h\right\rangle-\frac{1}{4}\left(|\bar{\nabla} h|^{2}+\left|\bar{\nabla}\left(\operatorname{tr}_{\bar{g}} h\right)\right|^{2}\right) \\
& +\frac{1}{2} h^{i j} h^{k l} \bar{R}_{i k j l}+E(h)+\bar{\nabla}_{i}\left(E_{1}^{i}(h)\right)
\end{aligned}
$$


where $E(h)$ is a function and $E_{1}(h)$ is a vector field on $\Omega$ satisfying

$$
|E(h)| \leq C\left(|h||\bar{\nabla} h|^{2}+|h|^{3}\right), \quad\left|E_{1}(h)\right| \leq C|h|^{2}|\bar{\nabla} h|
$$

for a positive constant $C$ depending only on $n$.

Proof. First note that

$$
-\bar{\nabla}_{i}\left[\bar{g}^{i k} \bar{g}^{j l}\left(\bar{\nabla}_{k} h_{j l}-\bar{\nabla}_{l} h_{j k}\right)\right]-\langle\operatorname{Ric}(\bar{g}), h\rangle=D R_{\bar{g}}(h)
$$

Suppose $g^{i k}=\bar{g}^{i k}+\tau^{i k}$. Then $\tau^{i k}=-h^{i k}+E_{2}^{i k}(h)$ where $h^{i k}=\bar{g}^{i j} h_{j l} \bar{g}^{l k}$ and $\left|E_{2}(h)\right| \leq C|h|^{2}$. Hence,

$$
g^{i k} g^{j l}-\bar{g}^{i k} \bar{g}^{j l}=-\bar{g}^{i k} h^{j l}-\bar{g}^{j l} h^{i k}+E_{3}^{i k j l}(h)
$$

where $\left|E_{3}(h)\right| \leq C|h|^{2}$. Therefore,

$$
\begin{aligned}
-\bar{\nabla}_{i} & {\left[\left(g^{i k} g^{j l}-\bar{g}^{i k} \bar{g}^{j l}\right)\left(\bar{\nabla}_{k} h_{j l}-\bar{\nabla}_{l} h_{j k}\right)\right] } \\
= & \bar{\nabla}_{i}\left[\left(\bar{g}^{i k} h^{j l}+\bar{g}^{j l} h^{i k}-E_{3}^{i k j l}(h)\right)\left(\bar{\nabla}_{k} h_{j l}-\bar{\nabla}_{l} h_{j k}\right)\right] \\
= & \frac{1}{2} \Delta_{\bar{g}}|h|^{2}+\left\langle h, \nabla^{2} \operatorname{tr}_{\bar{g}}(h)\right\rangle_{\bar{g}}-\operatorname{div}_{\bar{g}} \operatorname{div}_{\bar{g}}\left(h^{2}\right) \\
& \quad-\bar{\nabla}_{i}\left(E_{3}^{i k j l}\left(\bar{\nabla}_{k} h_{j l}-\bar{\nabla}_{l} h_{j k}\right)\right) .
\end{aligned}
$$

Applying the Ricci identity, one has

$$
\begin{aligned}
\frac{1}{2} \bar{g}^{i j} \bar{g}^{k l} \bar{g}^{p q} \bar{\nabla}_{i} h_{k p} \bar{\nabla}_{l} h_{j q}= & \frac{1}{2} \operatorname{div}_{\bar{g}} \operatorname{div}_{\bar{g}}\left(h^{2}\right)-\frac{1}{2}\left\langle\operatorname{Ric}(\bar{g}), h^{2}\right\rangle \\
& +\frac{1}{2} h^{i j} h^{k l} \bar{R}_{i k j l} .
\end{aligned}
$$

The lemma follows from Proposition 2.1, (2.2), (2.3) and (2.4).

Next, let $D H_{\bar{g}}(h)$ denote the linearization of the mean curvature at $\bar{g}$ along $h$. Proposition 2.2 implies

$$
D H_{\bar{g}}(h)=\frac{1}{2}\left[h(\bar{\nu}, \bar{\nu}) H(\bar{g})-\sum_{\alpha=1}^{n-1}\left(2 \bar{\nabla}_{e_{\alpha}} h\left(e_{\alpha}, \bar{\nu}\right)-\bar{\nabla}_{\bar{\nu}} h\left(e_{\alpha}, e_{\alpha}\right)\right)\right]
$$


For later use, we note the following equivalent expression of $D H_{\bar{g}}(h)$ (see $[13$, (34)] for instance)

$$
D H_{\bar{g}}(h)=\frac{1}{2}\left\{\left[d\left(\operatorname{tr}_{\bar{g}} h\right)-\operatorname{div}_{\bar{g}} h\right](\bar{\nu})-\operatorname{div}_{\Sigma} X\right\},
$$

where $X$ is the vector field on $\Sigma$ dual to the 1 -form $\left.h(\bar{\nu}, \cdot)\right|_{T \Sigma}$.

Let $D R_{\bar{g}}^{*}(\cdot)$ denote the formal $L^{2} \bar{g}$-adjoint of $D R_{\bar{g}}(\cdot)$, i.e.,

$$
D R_{\bar{g}}^{*}(\lambda)=-\left(\Delta_{\bar{g}} \lambda\right) \bar{g}+\nabla_{\bar{g}}^{2} \lambda-\lambda \operatorname{Ric}(\bar{g})
$$

where $\lambda$ is a function and $\nabla_{\bar{g}}^{2} \lambda$ denotes the Hessian of $\lambda$ with respect to $\bar{g}$. The content of the following lemma had been used in [13].

Lemma 2.2. Let $p$ be any smooth $(0,2)$ symmetric tensor on $\Omega$, then

$$
\begin{aligned}
\int_{\Omega} D R_{\bar{g}}(p) \lambda d \operatorname{vol}_{\bar{g}}= & \int_{\Omega}\left\langle D R_{\bar{g}}^{*}(\lambda), p\right\rangle d \operatorname{vol}_{\bar{g}}-\int_{\Sigma} 2 D H_{\bar{g}}(p) \lambda d \sigma_{\bar{g}} \\
& +\int_{\Sigma} \lambda_{\bar{\nu}}\left(\operatorname{tr}_{\bar{g}}(p)-p(\bar{\nu}, \bar{\nu})\right) d \sigma_{\bar{g}}
\end{aligned}
$$

where $\lambda_{\bar{\nu}}=\partial_{\bar{\nu}} \lambda$ denotes the directional derivative of $\lambda$ along $\bar{\nu}$.

Proof. Let $Y$ be the vector field on $\Sigma$ dual to the 1-form $\left.p(\bar{\nu}, \cdot)\right|_{T \Sigma}$. Integrating by parts, one has

$$
\begin{aligned}
\int_{\Omega} & D R_{\bar{g}}(p) \lambda d \operatorname{vol}_{\bar{g}}-\int_{\Omega}\left\langle D R_{\bar{g}}^{*}(\lambda), p\right\rangle d \operatorname{vol}_{\bar{g}} \\
= & \int_{\Sigma}-\lambda \partial_{\bar{\nu}}\left(\operatorname{tr}_{\bar{g}} p\right)+\left(\operatorname{tr}_{\bar{g}} p\right) \partial_{\bar{\nu}} \lambda+\lambda \operatorname{div}_{\bar{g}} p(\bar{\nu})-p(\bar{\nu}, \bar{\nabla} \lambda) d \sigma_{\bar{g}} \\
= & \int_{\Sigma} \lambda\left[-\partial_{\bar{\nu}}\left(\operatorname{tr}_{\bar{g}} p\right)+\operatorname{div}_{\bar{g}} p(\bar{\nu})\right]-\left\langle Y, \bar{\nabla}^{\Sigma} \lambda\right\rangle d \sigma_{\bar{g}} \\
& +\int_{\Sigma} \lambda_{\bar{\nu}}\left(\operatorname{tr}_{\bar{g}}(p)-p(\bar{\nu}, \bar{\nu})\right) d \sigma_{\bar{g}} \\
= & \int_{\Sigma} \lambda\left[-\partial_{\bar{\nu}}\left(\operatorname{tr}_{\bar{g}} p\right)+\operatorname{div}_{\bar{g}} p(\bar{\nu})+\operatorname{div}_{\Sigma} Y\right] d \sigma_{\bar{g}} \\
& +\int_{\Sigma} \lambda_{\bar{\nu}}\left(\operatorname{tr}_{\bar{g}}(p)-p(\bar{\nu}, \bar{\nu})\right) d \sigma_{\bar{g}}
\end{aligned}
$$

where $\bar{\nabla}^{\Sigma}(\cdot)$ denotes the gradient on $\Sigma$ with respect to the induced metric. From this and (2.6) the Lemma follows.

Using Lemma 2.2, we can estimate $\int_{\Omega}[R(g)-R(\bar{g})] \lambda d \operatorname{vol}_{\bar{g}}$. 
Proposition 2.3. Suppose $g$ and $\bar{g}$ induce the same metric on $\Sigma$ and $h$ satisfies $\operatorname{div}_{\bar{g}} h=0$. Given any $C^{2}$ function $\lambda$ on $\Omega$, one has

$$
\begin{aligned}
\int_{\Omega}[ & R(g)-R(\bar{g})] \lambda d \operatorname{vol}_{\bar{g}} \\
= & \int_{\Omega}\left\langle h, D R_{\bar{g}}^{*}(\lambda)\right\rangle d \operatorname{vol}_{\bar{g}}-\frac{1}{2} \int_{\Omega}\left\langle h^{2}, D R_{\bar{g}}^{*}(\lambda)\right\rangle d \operatorname{vol}_{\bar{g}} \\
& +\int_{\Omega}\left[\left(\operatorname{tr}_{\bar{g}} h\right)\left\langle h, \nabla_{\bar{g}}^{2} \lambda\right\rangle+\frac{1}{2} h^{i j} h^{k l} \bar{R}_{i k j l} \lambda-\frac{1}{4}\left(|\bar{\nabla} h|^{2}+\left|\bar{\nabla}\left(\operatorname{tr}_{\bar{g}} h\right)\right|^{2}\right) \lambda\right] d \operatorname{vol}_{\bar{g}} \\
& +\int_{\Sigma}\left[-\left(h_{n n}\right)^{2}-\frac{1}{2}|X|^{2}\right] \lambda_{; n} d \sigma_{\bar{g}}-\int_{\Sigma} h_{n n}\left\langle X, \bar{\nabla}^{\Sigma} \lambda\right\rangle d \sigma_{\bar{g}} \\
& +\int_{\Sigma}\left[-\frac{1}{2}\left(h_{n n}\right)^{2} H(\bar{g})-\frac{1}{2} \overline{\mathbb{I}}(X, X)-\frac{3}{2}|X|^{2} H(\bar{g})\right] \lambda d \sigma_{\bar{g}} \\
& -\int_{\Sigma}\left(2-2 \operatorname{tr}_{\bar{g}} h\right) D H_{\bar{g}}(h) \lambda d \sigma_{\bar{g}}+\int_{\Omega} E(h) \lambda d \operatorname{vol}_{\bar{g}} \\
& -\int_{\Omega} E_{1}^{i}(h) \bar{\nabla}_{i} \lambda d \operatorname{vol}_{\bar{g}}+\int_{\Sigma} F_{1}(h) \lambda d \sigma_{\bar{g}}
\end{aligned}
$$

where $\overline{\mathbb{I I}}$ is the second fundamental form of $\Sigma$ in $(\Omega, \bar{g})$ with respect to $\bar{\nu}, X$ is the vector field on $\Sigma$ that is dual to the 1 -form $\left.h(\bar{\nu}, \cdot)\right|_{T \Sigma}, E(h)$ and $E_{1}^{i}(h)$ are as in Lemma 2.1, and $F_{1}(h)$ is a function on $\Sigma$ satisfying

$$
\left|F_{1}(h)\right| \leq C|h|^{2}|\bar{\nabla} h|
$$

for a positive constant $C$ depending only on $n$.

Proof. By (2.8) with $p=h$, using the fact that $\left.h\right|_{T(\Sigma)}=0$, we have

$$
\int_{\Omega} D R_{\bar{g}}(h) \lambda d \operatorname{vol}_{\bar{g}}=\int_{\Omega}\left\langle D R_{\bar{g}}^{*}(\lambda), h\right\rangle d \operatorname{vol}_{\bar{g}}-\int_{\Sigma} 2 D H_{\bar{g}}(h) \lambda d \sigma_{\bar{g}} .
$$

By the second line in (2.9) with $p=h^{2}$, and integrating by parts, we also have

$$
\begin{aligned}
\int_{\Omega} & -\frac{\lambda}{2} D R_{\bar{g}}\left(h^{2}\right)+\lambda\left\langle h, \bar{\nabla}^{2} \operatorname{tr}_{\bar{g}} h\right\rangle d \operatorname{vol}_{\bar{g}} \\
& =\int_{\Omega}-\frac{1}{2}\left\langle D R_{\bar{g}}^{*}(\lambda), h^{2}\right\rangle+\operatorname{tr}_{\bar{g}} h\left\langle h, \bar{\nabla}^{2} \lambda\right\rangle d \operatorname{vol}_{\bar{g}}+\mathcal{B},
\end{aligned}
$$


where

$$
\begin{aligned}
\mathcal{B}= & \int_{\Sigma} \frac{1}{2}\left[\lambda \partial_{\bar{\nu}}\left(|h|^{2}\right)-|h|^{2} \partial_{\bar{\nu}} \lambda-\lambda\left(\operatorname{div}_{\bar{g}} h^{2}\right)(\bar{\nu})+\left(h^{2}\right)(\bar{\nu}, \bar{\nabla} \lambda)\right] d \sigma_{\bar{g}} \\
& +\int_{\Sigma}\left[\lambda h\left(\bar{\nu}, \bar{\nabla} \operatorname{tr}_{\bar{g}} h\right)-\operatorname{tr}_{\bar{g}} h h(\bar{\nu}, \bar{\nabla} \lambda)\right] d \sigma_{\bar{g}}
\end{aligned}
$$

To compute $\mathcal{B}$, let $\left\{e_{\alpha} \mid 1 \leq \alpha \leq n-1\right\}$ be an orthonormal frame on $\Sigma$ and let $e_{n}=\bar{\nu}$. Denote $\bar{\nabla}$ also by ";", thus $h_{i j ; k}=\bar{\nabla}_{k} h_{i j}$. The assumptions $\left.h\right|_{T \Sigma}=0$ and $\operatorname{div}_{\bar{g}} h=0$ imply the following facts on $\Sigma$ :

$$
\begin{gathered}
|h|^{2}=\left(h_{n n}\right)^{2}+2|X|^{2},\left(h^{2}\right)_{n n}=\left(h_{n n}\right)^{2}+|X|^{2},\left(h^{2}\right)_{n \alpha}=h_{n n} h_{n \alpha} \\
\left(h^{2}\right)(\bar{\nu}, \bar{\nabla} \lambda)=\left[\left(h_{n n}\right)^{2}+|X|^{2}\right] \lambda_{; n}+h_{n n}\left\langle X, \bar{\nabla}^{\Sigma} \lambda\right\rangle \\
h_{\beta \gamma ; \alpha}=h_{\beta n} \overline{\mathbb{I}}_{\gamma \alpha}+h_{n \gamma} \overline{\mathbb{I}}_{\beta \alpha} \\
h_{n n ; \alpha}=\left(\operatorname{tr}_{\bar{g}} h\right)_{; \alpha}-\sum_{\beta=1}^{n-1} h_{\beta \beta ; \alpha}=\left(\operatorname{tr}_{\bar{g}} h\right)_{; \alpha}-2 \overline{\mathbb{I}}\left(X, e_{\alpha}\right) \\
0=\left(\operatorname{div}_{\alpha}\right)_{\alpha}=h_{\alpha n ; n}+\sum_{\beta=1}^{n-1} h_{\alpha \beta ; \beta}=h_{\alpha n ; n}+h_{n \alpha} H(\bar{g})+\overline{\mathbb{I}}\left(X, e_{\alpha}\right) \\
0=\left(\operatorname{div}_{\bar{g}} h\right)_{n}=h_{n n ; n}+\sum_{\alpha=1}^{n-1} h_{n \alpha ; \alpha}=h_{n n ; n}+\operatorname{div}_{\Sigma} X+h_{n n} H(\bar{g}) \\
2 D H_{\bar{g}}(h)=\left(\operatorname{tr}_{\bar{g}} h\right)_{; n}-\operatorname{div}_{\Sigma} X
\end{gathered}
$$

where (2.19) follows from (2.6). By (2.16)-(2.18), we have

$$
\begin{aligned}
\partial_{\bar{\nu}}\left(|h|^{2}\right)-\left(\operatorname{div}_{\bar{g}} h^{2}\right)(\bar{\nu})= & 3 h_{n \alpha} h_{n \alpha ; n}+h_{n n} h_{n n ; n}-h_{n \alpha} h_{n n ; \alpha} \\
= & -\overline{\mathbb{I I}}(X, X)-3 H(\bar{g})|X|^{2}-H(\bar{g})\left(h_{n n}\right)^{2} \\
& -h_{n n} \operatorname{div}_{\Sigma} X-\left\langle X, \bar{\nabla}^{\Sigma} \operatorname{tr}_{\bar{g}} h\right\rangle .
\end{aligned}
$$

By (2.12), (2.13), (2.14), (2.20) and integration by parts, we have

$$
\begin{aligned}
\mathcal{B}= & \int_{\Sigma}\left[-\left(h_{n n}\right)^{2}-\frac{1}{2}|X|^{2}\right] \lambda_{; n}-\int_{\Sigma} h_{n n}\left\langle X, \bar{\nabla}^{\Sigma} \lambda\right\rangle \\
& +\int_{\Sigma}\left[-\frac{1}{2} \overline{\mathbb{I}}(X, X)-\frac{3}{2} H(\bar{g})|X|^{2}-\frac{1}{2} H(\bar{g})\left(h_{n n}\right)^{2}+2 h_{n n} D H_{\bar{g}}(h)\right] \lambda d \sigma_{\bar{g}} .
\end{aligned}
$$


Note that

$$
\int_{\Omega}\left(\bar{\nabla}_{i} E_{1}^{i}(h)\right) \lambda d \operatorname{vol}_{\bar{g}}=-\int_{\Omega} E_{1}^{i}(h) \bar{\nabla}_{i} \lambda d \operatorname{vol}_{\bar{g}}+\int_{\Sigma} \lambda F_{1}(h) d \sigma_{\bar{g}},
$$

where $\left|F_{1}(h)=\left\langle E_{1}(h), \bar{\nu}\right\rangle\right| \leq C|h|^{2}|\bar{\nabla} h|$. Proposition 2.3 now follows from Lemma 2.1, (2.10), (2.11), (2.21) and (2.22).

The formula (2.23) below is a general form of [5, Theorem 10], which Brendle and Marques derived for geodesic balls in $\mathbb{S}^{n}$.

Theorem 2.1. Suppose $g$ and $\bar{g}$ induce the same metric on $\Sigma$ and $h$ satisfies $\operatorname{div}_{\bar{g}} h=0$. Given any $C^{2}$ function $\lambda$ on $\Omega$, one has

$$
\begin{aligned}
\int_{\Omega}[ & R(g)-R(\bar{g})] \lambda d \operatorname{vol}_{\bar{g}}+\int_{\Sigma}\left(2-\operatorname{tr}_{\bar{g}} h\right)[H(g)-H(\bar{g})] \lambda d \sigma_{\bar{g}} \\
= & \int_{\Omega}\left\langle h, D R_{\bar{g}}^{*}(\lambda)\right\rangle d \operatorname{vol}_{\bar{g}}-\frac{1}{2} \int_{\Omega}\left\langle h^{2}, D R_{\bar{g}}^{*}(\lambda)\right\rangle d \operatorname{vol}_{\bar{g}} \\
& +\int_{\Omega}\left[\left(\operatorname{tr}_{\bar{g}} h\right)\left\langle h, \nabla_{\bar{g}}^{2} \lambda\right\rangle+\frac{1}{2} h^{i j} h^{k l} \bar{R}_{i k j l} \lambda-\frac{1}{4}\left(|\bar{\nabla} h|^{2}+\left|\bar{\nabla}\left(\operatorname{tr}_{\bar{g}} h\right)\right|^{2}\right) \lambda\right] d \operatorname{vol}_{\bar{g}} \\
& +\int_{\Sigma}\left[-\frac{1}{4}\left(h_{n n}\right)^{2} H(\bar{g})-\frac{1}{2}\left(\overline{\mathbb{I I}}(X, X)+H(\bar{g})|X|^{2}\right)\right] \lambda d \sigma_{\bar{g}} \\
& +\int_{\Sigma} \lambda_{; n}\left[-\left(h_{n n}\right)^{2}-\frac{1}{2}|X|^{2}\right] d \sigma_{\bar{g}}+\int_{\Sigma}(-1) h_{n n}\left\langle X, \bar{\nabla}^{\Sigma} \lambda\right\rangle d \sigma_{\bar{g}} \\
& +\int_{\Omega} E(h) \lambda d \operatorname{vol}_{\bar{g}}+\int_{\Omega} Z^{i}(h) \bar{\nabla}_{i} \lambda d \operatorname{vol}_{\bar{g}}+\int_{\Sigma} F(h) \lambda d \sigma_{\bar{g}},
\end{aligned}
$$

where $E(h)$ is a function and $Z(h)$ is a vector field on $\Omega$ satisfying

$$
|E(h)| \leq C\left(|h||\bar{\nabla} h|^{2}+|h|^{3}\right), \quad|Z(h)| \leq C|h|^{2}|\bar{\nabla} h|,
$$

and $F(h)$ is some function on $\Sigma$ satisfying

$$
|F(h)| \leq C\left(|h|^{2}|\bar{\nabla} h|+|h|^{3}\right)
$$

Proof. Proposition 2.2 implies

$$
2[H(g)-H(\bar{g})]=2 D H_{\bar{g}}(h)+J(h)+F_{2}(h)
$$


where

$$
J(h)=\left[\frac{1}{4}\left(h_{n n}\right)^{2}+|X|^{2}\right] H(\bar{g})-h_{n n} D H_{\bar{g}}(h)
$$

and $F_{2}(h)$ is some function on $\Sigma$ satisfying $\left|F_{2}(h)\right| \leq C\left(|h|^{2}|\bar{\nabla} h|+|h|^{3}\right)$. Therefore

$$
\begin{aligned}
\left(2-h_{n n}\right)[H(g)-H(\bar{g})]= & \left(2-2 h_{n n}\right) D H_{\bar{g}}(h) \\
& +\left[\frac{1}{4}\left(h_{n n}\right)^{2}+|X|^{2}\right] H(\bar{g}) \\
& +F_{2}(h)-\frac{1}{2} h_{n n}\left[J(h)+F_{2}(h)\right] .
\end{aligned}
$$

(2.23) now follows readily from Proposition 2.3 and (2.25).

The term $D R_{\bar{g}}^{*}(\lambda)$ in (2.23) may suggest that one consider a background metric $\bar{g}$ which admits a nontrivial function $\lambda$ such that $D R_{\bar{g}}^{*}(\lambda)=0$ (such metrics are known as static metrics [10].) For instance, if $\Omega$ is a geodesic ball $B$ in $\mathbb{S}^{n}, \bar{g}$ is the standard metric on $\mathbb{S}^{n}$ and $\lambda=\cos r$, where $r$ is the $\bar{g}$-distance to the center of $B$, then (2.23) reduces to the formula in [5, Theorem 10].

Besides static metrics, one can also consider those metrics $\bar{g}$ with the property that there exists a function $\lambda$ such that

$$
D R_{\bar{g}}^{*}(\lambda)=\bar{g}
$$

These metrics were studied by the authors in $[13,14]$. In this case, the terms

$$
\int_{\Omega}\left\langle h, D R_{\bar{g}}^{*}(\lambda)\right\rangle d \operatorname{vol}_{\bar{g}}-\frac{1}{2} \int_{\Omega}\left\langle h^{2}, D R_{\bar{g}}^{*}(\lambda)\right\rangle d \operatorname{vol}_{\bar{g}}
$$

in (2.23) become

$$
\int_{\Omega} \operatorname{tr}_{\bar{g}} h d \operatorname{vol}_{\bar{g}}-\frac{1}{2} \int_{\Omega}|h|^{2} d \operatorname{vol}_{\bar{g}} .
$$

To compensate these terms, one can include the difference between the volumes of $g$ and $\bar{g}$ into $(2.23)$.

Corollary 2.1. Suppose $\bar{g}$ is a metric on $\Omega$ with the property that there exists a function $\lambda$ satisfying $D R_{\bar{g}}^{*}(\lambda)=\bar{g}$. Let $g=\bar{g}+h$ be a nearby metric such that $g$ and $\bar{g}$ induce the same metric on $\Sigma$ and $h$ satisfies $\operatorname{div}_{\bar{g}} h=0$. 
Let $V(g), V(\bar{g})$ denote the volume of $(\Omega, g),(\Omega, \bar{g})$. Then

$$
\begin{aligned}
-2 & V(g)-V(\bar{g}))+\int_{\Omega}[R(g)-R(\bar{g})] \lambda d \operatorname{vol}_{\bar{g}} \\
& +\int_{\Sigma}\left(2-\operatorname{tr}_{\bar{g}} h\right)[H(g)-H(\bar{g})] \lambda d \sigma_{\bar{g}} \\
& =\int_{\Omega}\left[-\frac{1}{4}-\frac{1}{n-1}\right]\left(\operatorname{tr}_{\bar{g}} h\right)^{2} d \operatorname{vol}_{\bar{g}} \\
& +\int_{\Omega}\left[-\frac{1}{4}\left(|\bar{\nabla} h|^{2}+\left|\nabla_{\bar{g}}\left(\operatorname{tr}_{\bar{g}} h\right)\right|^{2}\right) \lambda\right] d \operatorname{vol}_{\bar{g}} \\
& \left.+\int_{\Omega}\left[\frac{1}{1-n} R(\bar{g})\left(\operatorname{tr}_{\bar{g}} h\right)^{2}+\left\langle h, \operatorname{Ric}_{\bar{g}}\right)\right\rangle\left(\operatorname{tr}_{\bar{g}} h\right)+\frac{1}{2} h_{i j} h_{k l} R_{i k j l}\right] \lambda d \operatorname{vol}_{\bar{g}} \\
& +\int_{\Sigma}\left[-\frac{1}{4}\left(h_{n n}\right)^{2} H(\bar{g})-\frac{1}{2}\left(\overline{\mathbb{I}}(X, X)+H(\bar{g})|X|^{2}\right)\right] \lambda d \sigma_{\bar{g}} \\
& +\int_{\Sigma} \lambda_{; n}\left[-\left(h_{n n}\right)^{2}-\frac{1}{2}|X|^{2}\right] d \sigma_{\bar{g}}+\int_{\Sigma}(-1) h_{n n}\left\langle X, \bar{\nabla}^{\Sigma} \lambda\right\rangle d \sigma_{\bar{g}} \\
& +\int_{\Omega} G(h) d \operatorname{vol}_{\bar{g}}+\int_{\Omega} E(h) \lambda d \operatorname{vol}_{\bar{g}}+\int_{\Omega} Z^{i}(h) \bar{\nabla}_{i} \lambda d \operatorname{vol}_{\bar{g}} \\
& +\int_{\Sigma} F(h) \lambda d \sigma_{\bar{g}},
\end{aligned}
$$

where $G(h)$ and $E(h)$ are functions on $\Omega$ satisfying

$$
|G(h)| \leq C|h|^{3}, \quad|E(h)| \leq C\left(|h||\bar{\nabla} h|^{2}+|h|^{3}\right),
$$

$Z(h)$ is a vector field on $\Omega$ satisfying

$$
|Z(h)| \leq C|h|^{2}|\bar{\nabla} h|
$$

and $F(h)$ is a function on $\Sigma$ satisfying

$$
|F(h)| \leq C\left(|h|^{2}|\bar{\nabla} h|+|h|^{3}\right) .
$$

Proof. The difference between the volumes of $\bar{g}$ and $g=\bar{g}+h$ is

$$
V(g)-V(\bar{g})=\int_{\Omega} \frac{1}{2}\left(\operatorname{tr}_{\bar{g}} h\right)+\left[\frac{1}{8}\left(\operatorname{tr}_{\bar{g}} h\right)^{2}-\frac{1}{4}|h|^{2}\right]+G(h) d \operatorname{vol}_{\bar{g}}
$$


where $G(h)$ is a function satisfying $|G(h)| \leq C|h|^{3}$ for a constant $C$ depending only on $n$. Suppose $D R_{\bar{g}}^{*}(\lambda)=\bar{g}$, i.e.,

$$
-\left(\Delta_{\bar{g}} \lambda\right) \bar{g}+\nabla_{\bar{g}}^{2} \lambda-\lambda \operatorname{Ric}(\bar{g})=\bar{g} .
$$

Taking trace, one has $\Delta_{\bar{g}} \lambda=\frac{1}{1-n}[R(\bar{g}) \lambda+n]$. Thus,

$$
\nabla_{\bar{g}}^{2} \lambda=\frac{1}{1-n}[R(\bar{g}) \lambda+1] \bar{g}+\lambda \operatorname{Ric}(\bar{g}) .
$$

(2.27) follows from (2.23), (2.28) and (2.29).

\section{Volume constrained rigidity}

We prove Theorem 1.3 in this section. First, we recall its statement:

Theorem 3.1. Let $(\Omega, \bar{g})$ be an n-dimensional compact Riemannian manifold, of constant sectional curvature 1 , with smooth boundary $\Sigma$. Suppose $\overline{\mathbb{I I}}+\bar{H} \bar{\gamma} \geq 0$ (i.e., $\overline{\mathbb{I I}}+\bar{H} \bar{\gamma}$ is positive semi-definite), where $\bar{\gamma}$ is the induced metric on $\Sigma$ and $\overline{\mathbb{I I}}, \bar{H}$ are the second fundamental form, the mean curvature of $\Sigma$ in $(\Omega, \bar{g})$. Suppose the first nonzero Neumann eigenvalue $\mu$ of $(\Omega, \bar{g})$ satisfies $\mu>n-\frac{2}{n+1}$.

Consider a nearby metric $g$ on $\Omega$ with the properties

- $R(g) \geq n(n-1)$ where $R(g)$ is the scalar curvature of $g$,

- $H(g) \geq \bar{H}$ where $H(g)$ is the mean curvature of $\Sigma$ in $(\Omega, g)$,

- $g$ and $\bar{g}$ induce the same metric on $\Sigma$,

- $V(g) \geq V(\bar{g})$ where $V(g), V(\bar{g})$ are the volumes of $g, \bar{g}$.

If $\|g-\bar{g}\|_{C^{2}(\bar{\Omega})}$ is sufficiently small, then there is a diffeomorphism $\varphi$ on $\Omega$ with $\left.\varphi\right|_{\Sigma}=\mathrm{id}$, which is the identity map on $\Sigma$, such that $\varphi^{*}(g)=\bar{g}$.

Proof. Fix a real number $p>n$. By [5, Proposition 11], if $\|g-\bar{g}\|_{W^{2, p}(\Omega)}$ is sufficiently small, there exists a $W^{3, p}$ diffeomorphism $\varphi$ on $\Omega$ with $\left.\varphi\right|_{\Sigma}=$ id such that $h=\varphi^{*}(g)-g$ is divergence free with respect to $\bar{g}$, and $\|h\|_{W^{2, p}(\Omega)} \leq N\|g-\bar{g}\|_{W^{2, p}(\Omega)}$ for some positive constant $N$ depending only on $(\Omega, \bar{g})$. Replacing $g$ by $\varphi^{*}(g)$, we may assume $g=\bar{g}+h$ with $\operatorname{div}_{\bar{g}} h=0$. We want to prove that if $\|h\|_{C^{1}(\bar{\Omega})}$ is sufficiently small and $g$ satisfies the conditions in the theorem, then $h$ must be zero. 
Since $\bar{g}$ has constant sectional curvature 1 , we choose $\lambda=-\frac{1}{n-1}$ such that $D R_{\bar{g}}^{*}(\lambda)=\bar{g}$. Corollary 2.1 then shows

$$
\begin{aligned}
& -2(V(g)-V(\bar{g}))-\frac{1}{n-1} \int_{\Omega}[R(g)-R(\bar{g})] d \operatorname{vol}_{\bar{g}} \\
& -\frac{1}{n-1} \int_{\Sigma}\left(2-\operatorname{tr}_{\bar{g}} h\right)[H(g)-H(\bar{g})] d \sigma_{\bar{g}} \\
& \geq \frac{1}{4(n-1)} \int_{\Omega}\left[-(n+1)\left(\operatorname{tr}_{\bar{g}} h\right)^{2}+2|h|^{2}+|\bar{\nabla} h|^{2}+\left|\bar{\nabla}\left(\operatorname{tr}_{\bar{g}} h\right)\right|^{2}\right] d \operatorname{vol}_{\bar{g}} \\
& \quad+\frac{1}{4(n-1)} \int_{\Sigma}\left[\left(h_{n n}\right)^{2} H(\bar{g})+2\left(\overline{\mathbb{I I}}(X, X)+H(\bar{g})|X|^{2}\right)\right] d \sigma_{\bar{g}} \\
& \quad-C|| h \|_{C^{1}(\bar{\Omega})}\left[\int_{\Omega}\left(|h|^{2}+|\bar{\nabla} h|^{2}\right) d \operatorname{vol}_{\bar{g}}+\int_{\Sigma}|h|^{2} d \sigma_{\bar{g}}\right]
\end{aligned}
$$

for a constant $C$ depending only on $(\Omega, \bar{g})$.

Using the variational property of $\mu$, we have

$$
\int_{\Omega}\left|\bar{\nabla}\left(\operatorname{tr}_{\bar{g}} h\right)\right|^{2} d \operatorname{vol}_{\bar{g}} \geq \mu\left[\left(\int_{\Omega}\left(\operatorname{tr}_{\bar{g}} h\right)^{2} d \operatorname{vol}_{\bar{g}}\right)-\frac{1}{V(\bar{g})}\left(\int_{\Omega} \operatorname{tr}_{\bar{g}} h d \operatorname{vol}_{\bar{g}}\right)^{2}\right] .
$$

By $(2.28), \int_{\Omega} \operatorname{tr}_{\bar{g}} h d \operatorname{vol}_{\bar{g}}$ is related to $(V(g)-V(\bar{g}))$ by

$$
\int_{\Omega} \operatorname{tr}_{\bar{g}} h d \operatorname{vol}_{\bar{g}}=2(V(g)-V(\bar{g}))-\int_{\Omega}\left\{\left[\frac{1}{4}\left(\operatorname{tr}_{\bar{g}} h\right)^{2}-\frac{1}{2}|h|^{2}\right]+2 G(h)\right\} d \operatorname{vol}_{\bar{g}},
$$

where $G(h) \leq C|h|^{3}$.

Given any constant $0<\epsilon<1$, using (3.2) and the fact $|h|^{2} \geq \frac{1}{n}\left(\operatorname{tr}_{\bar{g}} h\right)^{2}$ and $|\bar{\nabla} h|^{2} \geq \frac{1}{n}\left|\bar{\nabla}\left(\operatorname{tr}_{\bar{g}} h\right)\right|^{2}$, we have

$$
\begin{aligned}
\int_{\Omega} & {\left[-(n+1)\left(\operatorname{tr}_{\bar{g}} h\right)^{2}+2|h|^{2}+|\bar{\nabla} h|^{2}+\left|\nabla_{\bar{g}}\left(\operatorname{tr}_{\bar{g}} h\right)\right|^{2}\right] d \operatorname{vol}_{\bar{g}} } \\
\geq & \int_{\Omega}\left[\epsilon|h|^{2}+\epsilon|\bar{\nabla} h|^{2}+\left[-(n+1)+\frac{2-\epsilon}{n}\right]\left(\operatorname{tr}_{\bar{g}} h\right)^{2}\right. \\
& \left.+\left[\frac{(1-\epsilon)}{n}+1\right]\left|\bar{\nabla}\left(\operatorname{tr}_{\bar{g}} h\right)\right|^{2}\right] d \operatorname{vol}_{\bar{g}}
\end{aligned}
$$




$$
\begin{aligned}
& \geq \int_{\Omega}\left[\epsilon|h|^{2}+\epsilon|\bar{\nabla} h|^{2}+\left[-(n+1)+\frac{2-\epsilon}{n}+\frac{(1-\epsilon)}{n} \mu+\mu\right]\left(\operatorname{tr}_{\bar{g}} h\right)^{2}\right] d \operatorname{vol}_{\bar{g}} \\
& \quad-\mu\left[\frac{(1-\epsilon)}{n}+1\right] \frac{1}{V(\bar{g})}\left(\int_{\Omega} \operatorname{tr}_{\bar{g}} h d \operatorname{vol}_{\bar{g}}\right)^{2} .
\end{aligned}
$$

Since $\mu>n-\frac{2}{n+1}$, we can chose $\epsilon$ (depending only on $\mu$ and $n$ ) such that

$$
\left[-(n+1)+\frac{2-\epsilon}{n}+\frac{(1-\epsilon)}{n} \mu+\mu\right] \geq 0 .
$$

Then it follows from (3.3), (3.4) and (3.5) that

$$
\begin{aligned}
& \left.\left.\int_{\Omega}\left(-(n+1)\left(\operatorname{tr}_{\bar{g}} h\right)^{2}+2|h|^{2}+|\bar{\nabla} h|^{2}+\mid \bar{\nabla}\left(\operatorname{tr}_{\bar{g}} h\right)\right)\right|^{2}\right) d \operatorname{vol}_{\bar{g}} \\
& \quad \geq \epsilon \int_{\Omega}\left(|h|^{2}+|\bar{\nabla} h|^{2}\right) d \operatorname{vol}_{\bar{g}}-C_{1}(V(g)-V(\bar{g}))^{2}-C_{1} \int_{\Omega}|h|^{4} d \sigma_{\bar{g}}
\end{aligned}
$$

where $C_{1}$ is a positive constant depending only on $(\Omega, \bar{g})$.

At the boundary $\Sigma$, the assumption $\overline{\mathbb{I I I}}+H(\bar{g}) \bar{\gamma} \geq 0$ implies $H(\bar{g}) \geq 0$, therefore

$$
\int_{\Sigma}\left[\left(h_{n n}\right)^{2} H(\bar{g})+2\left(\overline{\mathbb{I}}(X, X)+H(\bar{g})|X|^{2}\right)\right] d \sigma_{\bar{g}} \geq 0
$$

for any $h$. By (3.1), (3.6) and (3.7), we have

$$
\begin{aligned}
& -8(n-1)(V(g)-V(\bar{g}))-4 \int_{\Omega}[R(g)-R(\bar{g})] d \operatorname{vol}_{\bar{g}} \\
& -4 \int_{\Sigma}\left(2-\operatorname{tr}_{\bar{g}} h\right)[H(g)-H(\bar{g})] d \sigma_{\bar{g}} \\
& \quad \geq \epsilon \int_{\Omega}\left(|h|^{2}+|\bar{\nabla} h|^{2}\right) d \operatorname{vol}_{\bar{g}} \\
& -C(V(g)-V(\bar{g}))^{2}-C \int_{\Omega}|h|^{4} d \operatorname{vol}_{\bar{g}} \\
& -\left.C|| h\right|_{C^{1}(\bar{\Omega})}\left[\int_{\Omega}\left(|h|^{2}+|\bar{\nabla} h|^{2}\right) d \operatorname{vol}_{\bar{g}}+\int_{\Sigma}|h|^{2} d \sigma_{\bar{g}}\right]
\end{aligned}
$$

for some positive constant $C$ depending only on $(\Omega, \bar{g})$. 
Finally, we note that

$$
(V(g)-V(\bar{g}))^{2} \leq C\left(\int_{\Omega}|h| d \operatorname{vol}_{\bar{g}}\right)(V(g)-V(\bar{g}))
$$

by (3.3) and the assumption $V(g) \geq V(\bar{g})$. Also, by the trace theorem,

$$
\|h\|_{L^{2}(\Sigma)} \leq C\|h\|_{W^{1,2}(\Omega)}
$$

for a constant $C$ only depending on $\Omega$. Therefore, by (3.8), (3.9), (3.10) and the assumptions $V(g) \geq V(\bar{g}), R(g) \geq R(\bar{g})$ and $H(g) \geq H(\bar{g})$, we conclude that if $\|h\|_{C^{1}(\bar{\Omega})}$ is sufficiently small, then

$$
0 \geq \frac{\epsilon}{2} \int_{\Omega}\left(|h|^{2}+|\bar{\nabla} h|^{2}\right) d \operatorname{vol}_{\bar{g}}
$$

which implies $h$ must be identically zero. This completes the proof.

Remark 3.1. In Theorem 3.1, if $\Sigma$ is indeed empty, i.e., $(\Omega, \bar{g})$ is a closed space form, its first nonzero Neumann eigenvalue satisfies $\mu \geq n$ as $(\Omega, \bar{g})$ is covered by $\mathbb{S}^{n}$. In this case, Theorem 3.1 says that $V(g) \geq V(\bar{g})$ implies $g$ is isometric to $\bar{g}$ for a nearby metrics $g$ with $R(g) \geq R(\bar{g})$. This could be compared to a more profound theorem known in three-dimension: "If $(M, g)$ is closed 3 -manifold with $R(g) \geq 6, \operatorname{Ric}(g) \geq g$ and $V(g) \geq V\left(\mathbb{S}^{3}\right)$, then $(M, g)$ is isometric to $\mathbb{S}^{3}$." (See [4, Corollary 5.4] and earlier reference of $[3,11])$

When $\Sigma \neq \emptyset$, the boundary assumption $\overline{\mathbb{I I}}+\bar{H} \bar{\gamma} \geq 0$ in Theorem 3.1 can be relaxed in certain circumstances. A detailed examination of the above proof shows, if

$$
\overline{\mathbb{I I}}(v, v)+\bar{H} \bar{\gamma} \geq-\beta \bar{\gamma}
$$

for some positive constant $\beta$, where $\beta$ is sufficiently small comparing to the constant $\epsilon$ in (3.5) and the constant $C$ in (3.10), then the conclusion of Theorem 3.1 still holds on such an $(\Omega, \bar{g})$. In particular, this shows

Corollary 3.1. Let $(M, \bar{g})$ be an $n$-dimensional Riemannian manifold of constant sectional curvature 1. Suppose $\Omega \subset M$ is a bounded domain with smooth boundary $\Sigma$, satisfying the assumptions in Theorem 3.1, i.e., $\mu>$ $n-\frac{2}{n+1}$ and $\overline{\mathbb{I I}}+\bar{H} \bar{\gamma} \geq 0$ on $\Sigma$. Let $\tilde{\Omega} \subset M$ be another bounded domain with smooth boundary $\tilde{\Sigma}$. If $\tilde{\Sigma}$ is sufficiently close to $\Sigma$ in the $C^{2}$ norm, then the conclusion of Theorem 3.1 holds on $\tilde{\Omega}$. 
It is known that the fist nonzero Neumann eigenvalue of $\mathbb{S}_{+}^{n}$ is $n$ (see $[9$, Theorem 3]). Therefore, Theorem 1.2 follows from Theorem 3.1. Moreover, by Corollary 3.1, Theorem 3.1 holds on a geodesic ball in $\mathbb{S}^{n}$ whose radius is slightly larger than $\frac{\pi}{2}$.

By the next lemma, we know Theorem 3.1 also holds on any geodesic ball in $\mathbb{S}^{n}$ that is strictly contained in $\mathbb{S}_{+}^{n}$.

Lemma 3.1. Let $B(\delta) \subset \mathbb{S}^{n}$ be a geodesic ball of radius $\delta$. Let $\mu(\delta)$ be the first nonzero Neumann eigenvalue of $B(\delta)$.

(i) $\mu(\delta)$ is a strictly decreasing function of $\delta$ on $\left(0, \frac{\pi}{2}\right]$.

(ii) For any $0<\delta<\frac{\pi}{2}$,

$$
\mu(\delta)>n+\frac{(\sin \delta)^{n-2} \cos \delta}{\int_{0}^{\delta}(\sin t)^{n-1} d t}>\frac{n}{(\sin \delta)^{2}} .
$$

Proof. By [9, Theorem 2, p.44], $\mu(\delta)$ is characterized by the fact that

$$
\left\{(\sin t)^{n-1} J^{\prime}\right\}^{\prime}+\left[\mu(\delta)-(n-1)(\sin t)^{-2}\right](\sin t)^{n-1} J=0
$$

has a solution $J=J(t)$ on $[0, \delta]$ satisfying

$$
J(0)=0, \quad J^{\prime}(\delta)=0, \quad J^{\prime}(t) \neq 0, \quad \forall t \in[0, \delta) .
$$

Given $0<\delta_{1}<\delta_{2} \leq \frac{\pi}{2}$, let $J_{i}=J_{i}(t)$ be a solution to (3.13) with $\mu(\delta)$ replaced by $\mu\left(\delta_{i}\right)$, satisfying (3.14) on $\left[0, \delta_{i}\right], i=1,2$. Replacing $J_{i}$ by $-J_{i}$ if necessary, we may assume that $J_{i}^{\prime}>0$ on $\left[0, \delta_{i}\right)$, hence $J_{i}>0$ on $\left(0, \delta_{i}\right]$. Define

$$
f_{i}=\frac{(\sin t)^{n-1} J_{i}^{\prime}}{J_{i}}, \quad \beta_{i}(t)=\left[\mu\left(\delta_{i}\right)-\frac{n-1}{(\sin t)^{2}}\right](\sin t)^{n-1} .
$$

By (3.13), $f_{i}$ satisfies

$$
f_{i}^{\prime}=-\beta_{i}-\frac{1}{(\sin t)^{n-1}} f_{i}^{2}
$$

Therefore, on $\left(0, \delta_{1}\right]$,

$$
\left(f_{1}-f_{2}\right)^{\prime}=\frac{1}{(\sin t)^{n-1}}\left(f_{2}^{2}-f_{1}^{2}\right)+\left[\mu\left(\delta_{2}\right)-\mu\left(\delta_{1}\right)\right](\sin t)^{n-1} .
$$

Note that $f_{1}(t), f_{2}(t)$ can be extended continuously to 0 such that $f_{1}(0)=$ $f_{2}(0)$. Moreover, $f_{1}>0, f_{2}>0$ on $\left(0, \delta_{1}\right), f_{2}\left(\delta_{1}\right)>0=f_{1}\left(\delta_{1}\right)$. Let $0 \leq t_{0}<$ 
$\delta_{1}$ be such that $f_{1}=f_{2}$ at $t_{0}$ and $f_{2}>f_{1}$ for $t_{0}<t \leq \delta_{1}$. On $\left(t_{0}, \delta_{1}\right]$, one would have $\left(f_{1}-f_{2}\right)^{\prime}>0$ if $\mu\left(\delta_{2}\right) \geq \mu\left(\delta_{1}\right)$, which is a contradiction to $f_{2}>$ $f_{1}$. Therefore, $\mu\left(\delta_{2}\right)<\mu\left(\delta_{1}\right)$. This proves (i).

To prove (ii), we further claim that $t_{0}=0$, i.e., $f_{2}>f_{1}$ on $\left(0, \delta_{1}\right]$. If not, there would be a nonpositive local minimum of $\left(f_{2}-f_{1}\right)$ at some $\tilde{t}_{0} \in\left(0, t_{0}\right]$. At $\tilde{t}_{0},(3.15)$ implies

$$
0=\left(f_{1}-f_{2}\right)^{\prime} \leq\left[\mu\left(\delta_{2}\right)-\mu\left(\delta_{1}\right)\right]\left(\sin \tilde{t}_{0}\right)^{n-1}<0
$$

because $0<f_{2}\left(\tilde{t}_{0}\right) \leq f_{1}\left(\tilde{t}_{0}\right)$ and $\mu\left(\delta_{2}\right)<\mu\left(\delta_{1}\right)$. Hence $f_{2}>f_{1}$ on $\left(0, \delta_{1}\right]$. Integrating $(3.15)$ on $\left[0, \delta_{1}\right]$, we have

$$
-f_{2}\left(\delta_{1}\right)=\int_{0}^{\delta_{1}}\left(f_{1}-f_{2}\right)^{\prime} d t>\left[\mu\left(\delta_{2}\right)-\mu\left(\delta_{1}\right)\right] \int_{0}^{\delta_{1}}(\sin t)^{n-1} d t .
$$

Therefore

$$
\mu\left(\delta_{1}\right)>\mu\left(\delta_{2}\right)+\frac{f_{2}\left(\delta_{1}\right)}{\int_{0}^{\delta_{1}}(\sin t)^{n-1} d t} .
$$

Now let $\delta_{1}=\delta \in\left(0, \frac{\pi}{2}\right)$ and $\delta_{2}=\pi / 2$. Applying the fact that $\mu\left(\frac{\pi}{2}\right)=n, J_{2}=$ $\sin t$, and

$$
f_{2}=(\sin t)^{n-2} \cos t
$$

we have

$$
\begin{aligned}
\mu(\delta) & >n+\frac{(\sin \delta)^{n-2} \cos \delta}{\int_{0}^{\delta}(\sin t)^{n-1} d t} \\
& >n+\frac{(\sin \delta)^{n-2} \cos ^{2} \delta}{\int_{0}^{\delta} \cos t(\sin t)^{n-1} d t} \\
& =\frac{n}{\sin ^{2} \delta} .
\end{aligned}
$$

Therefore, (ii) is proved.

\section{A volume estimate on domains in $\mathbb{R}^{n}$}

On $\mathbb{R}^{n}$, the standard Euclidean metric $\bar{g}$ satisfies $D R_{\bar{g}}^{*}(\lambda)=\bar{g}$ with

$$
\lambda(x)=-\frac{1}{2(n-1)}|x-a|^{2}+L
$$


where $|\cdot|$ denotes the Euclidean length, $a \in \mathbb{R}^{n}$ is any fixed point and $L$ is an arbitrary constant. In this section, we use this fact and Corollary 2.1 to prove Theorem 1.4 in the introduction. First we need some lemmas.

Lemma 4.1. On a compact Riemannian manifold $(\Omega, \bar{g})$ with smooth boundary $\Sigma$, there exists a positive constant $C$ depending only on $(\Omega, \bar{g})$ such that, for any Lipschitz function $\phi$ on $\Sigma$, there is an extension of $\phi$ to a Lipschitz function $\widetilde{\phi}$ on $\Omega$ such that

$$
\int_{\Omega}\left(|\widetilde{\phi}|^{2}+|\bar{\nabla} \widetilde{\phi}|^{2}\right) d \operatorname{vol}_{\bar{g}} \leq C \int_{\Sigma}\left(\phi^{2}+\left|\bar{\nabla}^{\Sigma} \phi\right|^{2}\right) d \sigma_{\bar{g}}
$$

where $\bar{\nabla}, \bar{\nabla}^{\Sigma}$ denote the gradient on $\Omega, \Sigma$ respectively.

Proof. Let $d(\cdot, \Sigma)$ be the distance to $\Sigma$. Let $\delta>0$ be a small constant such that the tubular neighborhood $U_{2 \delta}=\{x \in \Omega \mid d(x, \Sigma)<2 \delta\}$ can be parametrized by $F: \Sigma \times[0,2 \delta) \rightarrow U_{2 \delta}$, with $F(y, t)=\exp _{y}(t \nu(y))$ where $\exp _{y}(\cdot)$ is the exponential map at $y \in \Sigma$ and $\nu(y)$ is the inward unit normal at $y$. In $U_{2 \delta}$, the metric $\bar{g}$ takes the form $d t^{2}+\sigma^{t}$, where $\left\{\sigma^{t}\right\}_{0 \leq t<2 \delta}$ is a family of metrics on $\Sigma$. By choosing $\delta$ sufficiently small, one can assume $\sigma^{t}$ is equivalent to $\sigma^{0}$ in the sense that $\frac{1}{2} \leq \sigma^{t}(v, v) \leq 2$ for any tangent vector $v$ with $\sigma^{0}(v, v)=1, \forall 0 \leq t<2 \delta$.

Let $\rho=\rho(t)$ be a fixed smooth cut-off function on $[0, \infty)$ such that $0 \leq$ $\rho \leq 1, \rho(t)=1$ for $0 \leq t \leq \delta$ and $\rho(t)=0$ for $t \geq \frac{3}{2} \delta$. On $U_{2 \delta}$, consider the function $\widetilde{\phi}(y, t)=\phi(y) \rho(t)$. Since $\widetilde{\phi}$ is identically zero outside $U_{\frac{3}{2} \delta}=\{x \in$ $\left.\Omega \mid d(x, \Sigma)<\frac{3}{2} \delta\right\}, \tilde{\phi}$ can be viewed as an extension of $\phi$ on $\Omega$. For such an $\widetilde{\phi}$, one has

$$
\int_{\Omega}|\widetilde{\phi}|^{2} d \operatorname{vol}_{\bar{g}} \leq \int_{0}^{2 \delta}\left(\int_{\Sigma}|\phi|^{2} d \sigma^{t}\right) d t \leq C \delta \int_{\Sigma}|\phi|^{2} d \sigma_{\bar{g}}
$$

and

$$
\begin{aligned}
\int_{\Omega}|\bar{\nabla} \widetilde{\phi}|^{2} d \operatorname{vol}_{\bar{g}} & \leq 2 \int_{U_{2 \delta}}\left(|\bar{\nabla} \rho|^{2} \phi^{2}+|\bar{\nabla} \phi|^{2} \rho^{2}\right) d \operatorname{vol}_{\bar{g}} \\
& \leq C \delta \int_{\Sigma}|\phi|^{2} d \sigma_{\bar{g}}+2 \int_{0}^{2 \delta}\left(\int_{\Sigma}\left|\bar{\nabla}_{t}^{\Sigma} \phi\right|^{2} d \sigma^{t}\right) d t \\
& \leq C\left[\int_{\Sigma}|\phi|^{2} d \sigma_{\bar{g}}+\int_{\Sigma}\left|\bar{\nabla}^{\Sigma} \phi\right|^{2} d \sigma_{\bar{g}}\right],
\end{aligned}
$$

where $\bar{\nabla}_{t}^{\Sigma}$ denotes the gradient on $\left(\Sigma, \sigma^{t}\right)$ and $C$ is a positive constant depending only on $(\Omega, \bar{g})$. (4.2) now follows from (4.3) and (4.4). 
Lemma 4.2. On a compact Riemannian manifold $(\Omega, \bar{g})$ with smooth boundary $\Sigma$, there exists a positive constant $C$ depending only on $(\Omega, \bar{g})$ such that, for any smooth $(0,2)$ symmetric tensor $h$ on $\Omega$, one has

$$
\int_{\Omega}|h|^{3} d \operatorname{vol}_{\bar{g}} \leq C\left(\int_{\Sigma}|h|^{3} d \sigma_{\bar{g}}+\|h\|_{C^{2}(\Omega)} \int_{\Sigma}|h|^{2} d \sigma_{\bar{g}}+\int_{\Omega}|h||\bar{\nabla} h|^{2} d \operatorname{vol}_{\bar{g}}\right) .
$$

Proof. On $\Omega$, let $\phi=|h|^{\frac{3}{2}}$. By lemma 4.1, there exists a Lipschitz function $\widetilde{\phi}$ on $\Omega$ such that $\left.\widetilde{\phi}\right|_{\Sigma}=\left.\phi\right|_{\Sigma}$ and

$$
\int_{\Omega}\left(|\widetilde{\phi}|^{2}+|\bar{\nabla} \widetilde{\phi}|^{2}\right) d \operatorname{vol}_{\bar{g}} \leq C \int_{\Sigma}\left(\phi^{2}+\left|\bar{\nabla}^{\Sigma} \phi\right|^{2}\right) d \sigma_{\bar{g}}
$$

Let $\lambda_{1}>0$ be the first Dirichlet eigenvalue of $(\Omega, \bar{g})$, then

$$
\begin{aligned}
\int_{\Omega} \phi^{2} d \operatorname{vol}_{\bar{g}} & \leq 2 \int_{\Omega}\left[\widetilde{\phi}^{2}+(\phi-\widetilde{\phi})^{2}\right] d \operatorname{vol}_{\bar{g}} \\
& \leq 2 \int_{\Omega} \widetilde{\phi}^{2} d \operatorname{vol}_{\bar{g}}+2 \lambda_{1}^{-1} \int_{\Omega}|\bar{\nabla}(\phi-\widetilde{\phi})|^{2} d \operatorname{vol}_{\bar{g}} \\
& \leq C\left[\int_{\Sigma}\left(\phi^{2}+\left|\bar{\nabla}^{\Sigma} \phi\right|^{2}\right) d \sigma_{\bar{g}}+\int_{\Omega}|\bar{\nabla} \phi|^{2} d \operatorname{vol}_{\bar{g}}\right],
\end{aligned}
$$

where

$$
\int_{\Omega}|\bar{\nabla} \phi|^{2} d \operatorname{vol}_{\bar{g}}=\left.\left.\int_{\Omega}|\bar{\nabla}| h\right|^{\frac{3}{2}}\right|^{2} d \operatorname{vol}_{\bar{g}} \leq \frac{9}{4} \int_{\Omega}|h||\bar{\nabla} h|^{2} d \operatorname{vol}_{\bar{g}} .
$$

To handle the boundary term $\int_{\Sigma}\left|\bar{\nabla}^{\Sigma} \phi\right|^{2} d \sigma_{\bar{g}}$, given any constant $\epsilon>0$, one considers

$$
\int_{\Sigma}\left|\bar{\nabla}^{\Sigma}\left(|h|^{2}+\epsilon\right)^{\frac{3}{4}}\right|^{2} d \sigma_{\bar{g}}=-\int_{\Sigma}\left(|h|^{2}+\epsilon\right)^{\frac{3}{4}} \Delta_{\Sigma}\left(|h|^{2}+\epsilon\right)^{\frac{3}{4}} d \sigma_{\bar{g}}
$$

where $\Delta_{\Sigma}$ denotes the Laplacian on $\Sigma$. Let $\left\{e_{\alpha} \mid \alpha=1, \ldots, n-1\right\}$ be a local orthonormal frame on $\Sigma$ and $e_{n}$ be the outward unit normal to $\Sigma$. Let $\bar{H}$ be the mean curvature of $\Sigma$ with respect to $e_{n}$. Denote covariant differentiation 
$\Omega$ by ";". Let $i, j$ run through $\{1, \ldots, n\}$. One has

$$
\begin{aligned}
\Delta_{\Sigma}|h|^{2} & =\sum_{\alpha}\left(|h|^{2}\right)_{; \alpha \alpha}-\bar{H}\left(|h|^{2}\right)_{; n} \\
& =\sum_{\alpha, i, j} 2\left(h_{i j} h_{i j ; \alpha \alpha}+h_{i j ; \alpha}^{2}\right)-\bar{H} \sum_{i, j} 2 h_{i j} h_{i j ; n} \\
& \geq-C\|h\|_{C^{2}(\bar{\Omega})}|h| .
\end{aligned}
$$

Therefore,

$$
\begin{aligned}
\Delta_{\Sigma}\left(|h|^{2}+\epsilon\right)^{\frac{3}{4}} & =\frac{3}{4}\left(|h|^{2}+\epsilon\right)^{-\frac{1}{4}} \Delta_{\Sigma}|h|^{2}-\frac{3}{16}\left(|h|^{2}+\epsilon\right)^{-\left.\left.\frac{5}{4}\left|\bar{\nabla}^{\Sigma}\right| h\right|^{2}\right|^{2}} \\
& \geq-C|| h \|_{C^{2}(\bar{\Omega})}\left(|h|^{2}+\epsilon\right)^{-\frac{1}{4}}|h|-\left.\left.\frac{3}{16}\left(|h|^{2}+\epsilon\right)^{-\frac{5}{4}}\left|\bar{\nabla}^{\Sigma}\right| h\right|^{2}\right|^{2} .
\end{aligned}
$$

It follows from (4.8) and (4.10) that

$$
\begin{aligned}
\int_{\Sigma}\left|\bar{\nabla}^{\Sigma}\left(|h|^{2}+\epsilon\right)^{\frac{3}{4}}\right|^{2} d \sigma_{\bar{g}} \leq & \left.C|| h\right|_{C^{2}(\bar{\Omega})} \int_{\Sigma}\left(|h|^{2}+\epsilon\right)^{\frac{1}{2}}|h| d \sigma_{\bar{g}} \\
& +\frac{1}{3} \int_{\Sigma}\left|\bar{\nabla}^{\Sigma}\left(|h|^{2}+\epsilon\right)^{\frac{3}{4}}\right|^{2} d \sigma_{\bar{g}}
\end{aligned}
$$

Letting $\epsilon \rightarrow 0$, one has

$$
\left.\left.\int_{\Sigma}\left|\bar{\nabla}^{\Sigma}\right| h\right|^{\frac{3}{2}}\right|^{2} d \sigma_{\bar{g}} \leq C|| h \|_{C^{2}(\bar{\Omega})} \int_{\Sigma}|h|^{2} d \sigma_{\bar{g}} .
$$

(4.5) now follows from (4.6), (4.7) and (4.12).

We recall the statement of Theorem 1.4 and give its proof.

Theorem 4.1. Let $\Omega \subset \mathbb{R}^{n}$ be a bounded domain with smooth boundary $\Sigma$. Suppose $\overline{\mathbb{I I}}+\bar{H} \bar{\gamma}>0$ (i.e., $\overline{\mathbb{I I}}+\bar{H} \bar{\gamma}$ is positive definite), where $\overline{\mathbb{I I}}, \bar{H}$ are the second fundamental form, the mean curvature of $\Sigma$ in $\mathbb{R}^{n}$ and $\bar{\gamma}$ is the metric on $\Sigma$ induced from the Euclidean metric $\bar{g}$. Let $g$ be another metric on $\bar{\Omega}$ satisfying

- $g$ and $\bar{g}$ induce the same metric on $\Sigma$.

- $H(g) \geq \bar{H}$, where $H(g)$ is the mean curvature of $\Sigma$ in $(\Omega, g)$.

Given any point $a \in \mathbb{R}^{n}$, there exists a constant $\Lambda>\frac{\max _{q \in \bar{\Omega}}|q-a|^{2}}{4(n-1)}$, which depends only on $\Omega$ and $a$, such that if $\|g-\bar{g}\|_{C^{3}(\bar{\Omega})}$ is sufficiently small, 
then

$$
V(g)-V(\bar{g}) \geq \int_{\Omega} R(g) \Phi d \operatorname{vol}_{\bar{g}}
$$

where $\Phi=-\frac{1}{4(n-1)}|x-a|^{2}+\Lambda>0$ on $\bar{\Omega}$.

Proof. Fix a number $p>n$. By the proof of [5, Proposition 11], one knows if $\|g-\bar{g}\|_{W^{3, p}(\Omega)}$ is sufficiently small, then there exists a $W^{4, p}$ diffeomorphism $\varphi: \Omega \rightarrow \Omega$ such that $\left.\varphi\right|_{\Sigma}=\mathrm{id}, h=\varphi^{*}(g)-\bar{g}$ is divergence free with respect to $\bar{g}$, and $\|h\|_{W^{3, p}(\Omega)} \leq N\|g-\bar{g}\|_{W^{3, p}(\Omega)}$ for a positive constant $N$ depending only on $(\Omega, \bar{g})$. In what follows, we will work with $\phi^{*}(g)$. For convenience, we still denote $\phi^{*}(g)$ by $g$.

Given $a \in \mathbb{R}^{n}$, consider $\lambda(x)=-\frac{1}{2(n-1)}|x-a|^{2}+L$ where $L$ is a constant to be determined. First, we require $L>\frac{1}{2(n-1)} \max _{q \in \bar{\Omega}}|q-a|^{2}$ so that $\lambda>0$ on $\bar{\Omega}$. Since $\lambda$ satisfies $D R_{\bar{g}}^{*}(\lambda)=\bar{g}$, Corollary 2.1 shows

$$
\begin{aligned}
-2(V(g)-V(\bar{g}))+\int_{\Omega} R(g) \lambda d \operatorname{vol}_{\bar{g}}+\int_{\Sigma}\left(2-\operatorname{tr}_{\bar{g}} h\right)[H(g)-\bar{H}] \lambda d \sigma_{\bar{g}} \\
\leq-\int_{\Omega} \frac{1}{4}|\bar{\nabla} h|^{2} \lambda d \operatorname{vol}_{\bar{g}}+\int_{\Sigma}\left[-\frac{1}{4}\left(h_{n n}\right)^{2} \bar{H}-\frac{1}{2}\left(\overline{\mathbb{I I}}(X, X)+\bar{H}|X|^{2}\right)\right] \lambda d \sigma_{\bar{g}} \\
\quad+\int_{\Sigma} \lambda_{; n}\left[-\left(h_{n n}\right)^{2}-\frac{1}{2}|X|^{2}\right] d \sigma_{\bar{g}}+\int_{\Sigma}(-1) h_{n n}\left\langle X, \bar{\nabla}^{\Sigma} \lambda\right\rangle d \sigma_{\bar{g}} \\
\quad+\int_{\Omega} G(h) d \operatorname{vol}_{\bar{g}}+\int_{\Omega} E(h) \lambda d \operatorname{vol}_{\bar{g}}+\int_{\Omega} Z^{i}(h) \bar{\nabla}_{i} \lambda d \operatorname{vol}_{\bar{g}} \\
\quad+\int_{\Sigma} F(h) \lambda d \sigma_{\bar{g}},
\end{aligned}
$$

where $\quad|G(h)| \leq C|h|^{3}, \quad|E(h)| \leq C\left(|h||\bar{\nabla} h|^{2}+|h|^{3}\right), \quad|Z(h)| \leq C|h|^{2}|\bar{\nabla} h|$, $|F(h)| \leq C\left(|h|^{2}|\bar{\nabla} h|+|h|^{3}\right)$ for some constant $C$ depending only on $\Omega$.

At $\Sigma, \lambda_{; n}$ and $\bar{\nabla}^{\Sigma} \lambda$ are determined solely by $\Omega$ and $a$ (in particular they are independent on $L$ ). Apply the assumption $\overline{\mathrm{III}}+\bar{H} \bar{\gamma}>0$ (which implies $\bar{H}>0)$ and the fact $|h|^{2}=\left(h_{n n}\right)^{2}+2|X|^{2}$, we have

$$
\begin{aligned}
& {\left[-\frac{1}{4}\left(h_{n n}\right)^{2} \bar{H}-\frac{1}{2}\left(\overline{\mathbb{I I}}(X, X)+\bar{H}|X|^{2}\right)\right] \lambda} \\
& \quad+\lambda_{; n}\left[-\left(h_{n n}\right)^{2}-\frac{1}{2}|X|^{2}\right]+(-1) h_{n n}\left\langle X, \bar{\nabla}^{\Sigma} \lambda\right\rangle \\
& \quad \leq-L C_{1}|h|^{2}+C_{2}|h|^{2},
\end{aligned}
$$


where $C_{1}, C_{2}$ are positive constants depending only on $\Omega$ and $a$. We fix $L$ such that

$$
L C_{1}-C_{2}>0
$$

and let $m=\frac{1}{4} \min _{\bar{\Omega}} \lambda$ (note that $\lambda$ is fixed now). (4.14)-(4.16) imply

$$
\begin{aligned}
& -2(V(g)-V(\bar{g}))+\int_{\Omega} R(g) \lambda d \operatorname{vol}_{\bar{g}}+\int_{\Sigma}\left(2-\operatorname{tr}_{\bar{g}} h\right)[H(g)-\bar{H}] \lambda d \sigma_{\bar{g}} \\
& \leq-m \int_{\Omega}|\bar{\nabla} h|^{2} d \operatorname{vol}_{\bar{g}}-\left(L C_{1}-C_{2}\right) \int_{\Sigma}|h|^{2} d \sigma_{\bar{g}} \\
& \quad+C_{3}\left(\int_{\Omega}\left(|h||\bar{\nabla} h|^{2}+|h|^{3}\right) d \operatorname{vol}_{\bar{g}}+\int_{\Sigma}\left(|h|^{2}|\bar{\nabla} h|+|h|^{3}\right) d \sigma_{\bar{g}}\right),
\end{aligned}
$$

where $C_{3}$ depends only on $\Omega, a$ and $L$. Apply Lemma 4.2 to the term $\int_{\Omega}|h|^{3} d \operatorname{vol}_{\bar{g}}$ on the right side of (4.17), we have

$$
\begin{aligned}
& -2(V(g)-V(\bar{g}))+\int_{\Omega} R(g) \lambda d \operatorname{vol}_{\bar{g}}+\int_{\Sigma}\left(2-\operatorname{tr}_{\bar{g}} h\right)[H(g)-\bar{H}] \lambda d \sigma_{\bar{g}} \\
& \leq-m \int_{\Omega}|\bar{\nabla} h|^{2} d \operatorname{vol}_{\bar{g}}-\left(L C_{1}-C_{2}\right) \int_{\Sigma}|h|^{2} d \sigma_{\bar{g}} \\
& \quad+C\|h\|_{C^{2}(\bar{\Omega})}\left(\int_{\Omega}|\bar{\nabla} h|^{2} d \operatorname{vol}_{\bar{g}}+\int_{\Sigma}|h|^{2} d \sigma_{\bar{g}}\right),
\end{aligned}
$$

where $C$ is independent on $h$. From this, we conclude that if $\|h\|_{C^{2}(\bar{\Omega})}$ is sufficiently small, then (4.13) holds with $\Phi=\frac{1}{2} \lambda$. This completes the proof.

Remark 4.1. When $\Omega \subset \mathbb{R}^{n}$ is a ball of radius $R$, one can take $a$ to be the center of $\Omega$. In this case, by computing $\bar{H}, \overline{\mathbb{I I}}$ and $\lambda_{; n}$ explicitly in (4.16), the constant $L$ can be chosen to be any constant satisfying

$$
L>\left[\frac{1}{2(n-1)}+\frac{4}{(n-1)^{2}}\right] R^{2} .
$$

Remark 4.2. By the results in $[12,17]$ based on the positive mass theorem $[16,18]$, a metric $g$ on $\Omega$ satisfying the boundary conditions in Theorem 4.1 must be isometric to the Euclidean metric if $R(g) \geq 0$. Therefore, a nontrivial metric $g$ in Theorem 4.1 necessarily has negative scalar curvature somewhere. For such a $g$, Theorem 4.1 shows if the weighted integral $\int_{\Omega} R(g) \Phi d \operatorname{vol}_{\bar{g}}$ is nonnegative, then $V(g) \geq V(\bar{g})$. 


\section{Other related results}

In this section, we collect some other by-products of the formulas derived in Section 2. First, we discuss a scalar curvature rigidity result for general domains in $\mathbb{S}^{n}$.

Theorem 5.1. Let $\Omega \subset \mathbb{S}^{n}$ be a smooth domain contained in a geodesic ball $B$ of radius less than $\frac{\pi}{2}$. Let $\bar{g}$ be the standard metric on $\mathbb{S}^{n}$. Let $\overline{\mathbb{I}}, \bar{H}$ be the second fundamental form, the mean curvature of $\Sigma=\partial \Omega$ in $(\Omega, \bar{g})$ with respect to the outward unit normal $\bar{\nu}$. Suppose $\overline{\mathbb{I I}} \geq-c \bar{\gamma}$, where $c \geq 0$ is a function on $\Sigma$ and $\bar{\gamma}$ is the induced metric on $\Sigma$. Let $q$ be the center of $B$. Suppose at $\Sigma \backslash\{q\}$,

$$
\bar{H}-c \geq\left[\frac{5 \cos \theta+\sqrt{\cos ^{2} \theta+8}}{2}\right] \tan r,
$$

where $r$ is the $\bar{g}$-distance to $q$ and $\theta$ is the angle between $\bar{\nu}$ and $\bar{\nabla} r$. Then the conclusion of Theorem 1.6 holds on $\Omega$.

Proof. As before, replacing $g$ by $\varphi^{*}(g)$ for some diffeomorphism $\varphi$, we may assume $\operatorname{div}_{\bar{g}} h=0$ where $h=g-\bar{g}$. On $\Omega$, let $\lambda=\cos r>0$, where $r$ is the $\bar{g}$-distance to $q$. At $\Sigma \backslash\{q\}$, we have

$$
\lambda_{; n}=-\sin r \cos \theta, \quad\left|\bar{\nabla}^{\Sigma} \lambda\right|=\sin r \sin \theta .
$$

Apply Theorem 2.1, using the fact $D R_{\bar{g}}^{*}(\lambda)=0$ and the assumptions on $R(g)$ and $H(g)$, we have

$$
\begin{aligned}
\int_{\Omega} & \left.\frac{1}{4}\left(|\bar{\nabla} h|^{2}+\left|\bar{\nabla}\left(\operatorname{tr}_{\bar{g}} h\right)\right|^{2}\right)+\frac{1}{2}\left(|h|^{2}+\left(\operatorname{tr}_{\bar{g}} h\right)^{2}\right)\right] \cos r d \operatorname{vol}_{\bar{g}} \\
\leq & \int_{\Sigma}\left[-\frac{1}{4}\left(h_{n n}\right)^{2} \bar{H}-\frac{1}{2}\left(\overline{\mathbb{I}}(X, X)+\bar{H}|X|^{2}\right)\right] \cos r d \sigma_{\bar{g}} \\
& +\int_{\Sigma \backslash\{q\}}\left[\left(h_{n n}\right)^{2}+\frac{1}{2}|X|^{2}\right](\sin r \cos \theta) d \sigma_{\bar{g}} \\
& +\int_{\Sigma \backslash\{q\}}\left|h_{n n} \| X\right|(\sin r \sin \theta) d \sigma_{\bar{g}} \\
& +\left.C|| h\right|_{C^{1}(\bar{\Omega})}\left\{\int_{\Omega}\left(|h|^{2}+|\bar{\nabla} h|^{2}\right) d \operatorname{vol}_{\bar{g}}+\int_{\Sigma}|h|^{2} d \sigma_{\bar{g}}\right\}
\end{aligned}
$$




$$
\begin{aligned}
\leq & -\int_{\Sigma \backslash\{q\}}\left[\left(\frac{1}{4}(\bar{H}-c) \cos r-\sin r \cos \theta\right)\left(h_{n n}\right)^{2}\right. \\
& +\frac{1}{2}((\bar{H}-c) \cos r-\sin r \cos \theta)|X|^{2} \\
& \left.-\left|h_{n n}\right||X|(\sin r \sin \theta)\right] d \sigma_{\bar{g}} \\
& +C|| h \|_{C^{1}(\bar{\Omega})}\left\{\int_{\Omega}\left(|h|^{2}+|\bar{\nabla} h|^{2}\right) d \operatorname{vol}_{\bar{g}}+\int_{\Sigma}|h|^{2} d \sigma_{\bar{g}}\right\}
\end{aligned}
$$

for some positive constant $C$ independent on $h$.

Note that the assumption (5.1) implies

$$
\frac{1}{4}(\bar{H}-c) \cos r-(\sin r \cos \theta) \geq 0
$$

and

$$
(\bar{H}-c) \cos r-(\sin r \cos \theta) \geq 0 \text {. }
$$

By (5.1), (5.4) and (5.5), we have

$$
\begin{aligned}
0 \leq & \left(\frac{1}{4}(\bar{H}-c) \cos r-\sin r \cos \theta\right)\left(h_{n n}\right)^{2}-\left|h_{n n}\right||X|(\sin r \sin \theta) \\
& +\frac{1}{2}((\bar{H}-c) \cos r-\sin r \cos \theta)|X|^{2}
\end{aligned}
$$

for any $h_{n n}$ and $X$. The result now follows from (5.3) and (5.6).

Remark 5.1. It is clear from the proof of Theorem 5.1 that the center $q$ of $B$ does not need to be inside $\Omega$.

Theorem 5.1 directly implies Theorem 1.7 in the introduction.

Proof of Theorem 1.7. Choose $c=0$ in Theorem 5.1. Since

$$
4 \geq \frac{5 \cos \theta+\sqrt{\cos ^{2} \theta+8}}{2}
$$

for any $\theta$, the result follows from Theorem 5.1.

Next, we consider a corresponding scalar curvature rigidity result when the background metric $\bar{g}$ is a flat metric. 
Theorem 5.2. Let $\Omega$ be a compact manifold with smooth boundary $\Sigma$. Suppose $\bar{g}$ is a smooth Riemannian metric on $\Omega$ such that $\bar{g}$ has zero sectional curvature and $\overline{\mathbb{I I}}+\bar{H} \bar{\gamma} \geq 0$ on $\Sigma$, where $\overline{\mathbb{I I}}, \bar{H}$ are the second fundamental form, the mean curvature of $\Sigma$, and $\bar{\gamma}$ is the induced metric on $\Sigma$. Suppose $g$ is another metric on $\Omega$ satisfying

- $R(g) \geq 0$ where $R(g)$ is the scalar curvature of $g$,

- $g$ and $\bar{g}$ induce the same metric on $\Sigma$,

- $H(g) \geq \bar{H}$ where $H(g)$ is the mean curvature of $\Sigma$ in $(\Omega, g)$.

If $\|g-\bar{g}\|_{C^{2}(\bar{\Omega})}$ is sufficiently small, then there is a diffeomorphism $\varphi$ on $\Omega$ with $\left.\varphi\right|_{\Sigma}=$ id such that $\varphi^{*}(g)=\bar{g}$.

Proof. As before, we may assume $\operatorname{div}_{\bar{g}} h=0$ where $h=g-\bar{g}$. Choose $\lambda=1$ in (2.23), one has

$$
\begin{aligned}
& \int_{\Omega}\left[\frac{1}{4}\left(|\bar{\nabla} h|^{2}+\left|\bar{\nabla}\left(\operatorname{tr}_{\bar{g}} h\right)\right|^{2}\right)\right] d \operatorname{vol}_{\bar{g}} \\
& \quad+\int_{\Sigma}\left[\frac{1}{4}\left(h_{n n}\right)^{2} H(\bar{g})+\frac{1}{2}\left(\overline{\mathbb{I}}(X, X)+H(\bar{g})|X|^{2}\right)\right] d \sigma_{\bar{g}} \\
& \quad \leq \int_{\Omega} E(h) d \operatorname{vol}_{\bar{g}}+\int_{\Sigma} F(h) d \sigma_{\bar{g}},
\end{aligned}
$$

where $|F(h)| \leq C\left(|h|^{2}|\bar{\nabla} h|+|h|^{3}\right)$ and $|E(h)| \leq C|h||\bar{\nabla} h|^{2}$ by Remark 2.1. The result follows from (5.7).

To finish, we mention that the positive Gaussian curvature condition of the boundary surface in [17] is not a necessary condition for the positivity of its Brown-York mass.

Theorem 5.3. Let $\Sigma \subset \mathbb{R}^{n}$ be a connected, closed hypersurface satisfying $\overline{\mathbb{I I}}+\bar{H} \bar{\gamma} \geq 0$, where $\overline{\mathbb{I I}}, \bar{H}$ are the second fundamental form, the mean curvature of $\Sigma$, and $\bar{\gamma}$ is the induced metric on $\Sigma$. Let $\Omega$ be the domain enclosed by $\Sigma$ in $\mathbb{R}^{n}$. Let $h$ be any nontrivial $(0,2)$ symmetric tensor on $\Omega$ satisfying

$$
\operatorname{div}_{\bar{g}} h=0, \quad \operatorname{tr}_{\bar{g}} h=0,\left.\quad h\right|_{T \Sigma}=0 .
$$

Let $\{g(t)\}_{|t|<\epsilon}$ be a 1-parameter family of metrics on $\Omega$ satisfying

$$
g(0)=\bar{g}, \quad g^{\prime}(0)=h, \quad R(g(t)) \geq 0,\left.\quad g(t)\right|_{T \Sigma}=\left.\bar{g}\right|_{T \Sigma} .
$$


Then

$$
\left.\int_{\Sigma} \bar{H} d \sigma_{\bar{g}}>\int_{\Sigma} H(g(t))\right) d \sigma_{\bar{g}}
$$

for small $t \neq 0$, where $H(g(t))$ is the mean curvature of $\Sigma$ in $(\Omega, g(t))$.

Proof. By Lemma 2.2, one knows

$$
\left.\frac{d}{d t}\left(\int_{\Omega}[R(g(t))-R(\bar{g})] d \operatorname{vol}_{\bar{g}}-2 \int_{\Sigma}[\bar{H}-H(g(t))] d \sigma_{\bar{g}}\right)\right|_{t=0}=0 .
$$

Direct calculation using Lemma 2.2, (2.17) and (5.8) shows

$$
\begin{gathered}
\left.\frac{d^{2}}{d t^{2}}\left(\int_{\Omega}[R(g(t))-R(\bar{g})] d \operatorname{vol}_{\bar{g}}-2 \int_{\Sigma}[\bar{H}-H(g(t))] d \sigma_{\bar{g}}\right)\right|_{t=0} \\
\quad=-\frac{1}{2} \int_{\Omega}|\bar{\nabla} h|^{2} d \operatorname{vol}_{\bar{g}}-\int_{\Sigma}\left[\left(\overline{\mathbb{I I}}(X, X)+H(\bar{g})|X|^{2}\right)\right] d \sigma_{\bar{g}}
\end{gathered}
$$

which is negative by the assumption on $\overline{\mathbb{I I}}+\bar{H} \bar{\gamma}$. Thus, for small $t$,

$$
2 \int_{\Sigma}[\bar{H}-H(g(t))] d \sigma_{\bar{g}}>\int_{\Omega}[R(g(t))-R(\bar{g})] d \operatorname{vol}_{\bar{g}} \geq 0
$$

Given an $h$ satisfying (5.8), a family of deformation $\{g(t)\}$ satisfying (5.9) is given by $g(t)=u(t)^{\frac{4}{n-2}}(\bar{g}+t h)$ for small $t$, where $u(t)>0$ is a conformal factor such that $R(g(t))=0$ (see [13, Lemma 4]).

An example of a non-convex surface $\Sigma \subset \mathbb{R}^{3}$, which is topologically a 2-sphere and satisfies the condition $\overline{\mathbb{I I}}+\bar{H} \bar{\gamma} \geq 0$, is given by a capsuleshaped surface with its middle slightly pinched.

\section{Acknowledgments}

P.M.'s work was partially supported by Australian Research Council Discovery Grant no. DP0987650 and by a 2011 Provost Research Award of the University of Miami. L.-F.T.'s work was partially supported by Hong Kong RGC General Research Fund no. CUHK 403011. The authors want to thank Simon Brendle and Fernando Marques for pointing out a false conjecture in a previous draft. The authors also want to thank the referees for useful comments that motivate Theorems 1.7 and 5.1. 


\section{References}

[1] R. Arnowitt, S. Deser and C.W. Misner, Coordinate invariance and energy expressions in general relativity, Phys. Rev. 122(2) (1961), 997-1006.

[2] R. Bartnik, The mass of an asymptotically flat manifold, Comm. Pure Appl. Math. 39 (1986), 661-693.

[3] H. Bray, The Penrose inequality in generla relativity and volume comparison theorems involving scalar curvature, $\mathrm{PhD}$ thesis, Standford University, 1997.

[4] S. Brendle, Rigidity phenomena involving scalar curvature, arXiv:1008.3097v2, to appear in Surveys in Differential Geometry.

[5] S. Brendle and F.C. Marques, Scalar curvature rigidity of geodesic balls in $\mathbb{S}^{n}$, J. Differ. Geom. 88 (2011), 379-394.

[6] S. Brendle, F.C. Marques and A. Neves, Deformations of the hemisphere that increase scalar curvature, Invent. Math. 185 (2011), 175-197.

[7] J.D. Brown and J.W. York Jr., Quasilocal energy in general relativity, in Mathematical aspects of classical field theory (Seattle, WA, 1991), Contemp. Math. 132, Amer. Math. Soc., Providence, RI, (1992), 129-142.

[8] J.D. Brown and J.W. York Jr., Quasilocal energy and conserved charges derived from the gravitational action, Phys. Rev. D (3), 47(4) (1993), 1407-1419.

[9] I. Chavel, Eigenvalue in Riemannian geometry, in Pure and Applied mathematics 115, Academic Press, (1984).

[10] J. Corvino, Scalar curvature deformation and a gluing construction for the Einstein constraint equations, Commun. Math. Phys. 214(1) (2000), $137-189$.

[11] M.J. Gursky and J.A. Viaclovsky, Volume comparison and the $\sigma_{k}$-Yamabe problem, Adv. Math. 187(2) (2004), 447-487.

[12] P. Miao, Positive mass theorem on manifolds admitting corners along a hypersurface, Adv. Theor. Math. Phys. 6(6) (2002), 1163-1182.

[13] P. Miao and L.-F. Tam, On the volume functional of compact manifolds with boundary with constant scalar curvature, Calc. Var. 36 (2009), 141-171. 
[14] P. Miao and L.-F. Tam, Einstein and conformally flat critical metrics of the volume functional, Trans. Amer. Math. Soc. 363(6) (2011), 2907-2937.

[15] M. Min-Oo, Scalar curvature rigidity of certain symmetric spaces, in Geometry, topology, and dynamics (Montreal, 1995), CRM Proc. Lecture Notes vol. 15, Amer. Math. Soc., Providence, RI, (1998), 127-137.

[16] R. Schoen and S.-T. Yau, On the proof of the positive mass conjecture in general relativity, Comm. Math. Phys. 65 (1979), 45-76.

[17] Y.-G. Shi and L.-F. Tam, Positive mass theorem and the boundary behaviors of compact manifolds with nonnegative scalar curvature, J. Differ. Geom. 62 (2002), 79-125.

[18] E. Witten, A new proof of the positive energy theorem, Commun. Math. Phys. 80 (1981), 381-402.

School of Mathematical Sciences

MONASH UNIVERSITY

VICTORIA 3800

Australia AND

DePARTMENT OF MATHEMATICS

UNIVERSITY OF MIAMI

Coral Gables

FL 33124

USA

E-mail address: Pengzi.Miao@sci.monash.edu.au; pengzim@math.miami.edu

The Institute of Mathematical Sciences and

Department of MATHEMATICS

The Chinese University of Hong Kong

Shatin

HONG KONG

CHINA

E-mail address: lftam@math.cuhk.edu.hk

Received September 14, 2011 